\title{
Diapycnal mixing in layered stratified plane Couette flow quantified in a tracer-based coordinate
}

\author{
Qi Zhou ${ }^{1} \dagger$, J. R. Taylor ${ }^{1}$, C. P. Caulfield ${ }^{2,1}$ and P. F. Linden ${ }^{1}$ \\ ${ }^{1}$ Department of Applied Mathematics and Theoretical Physics, University of Cambridge, \\ Wilberforce Road, Cambridge CB3 0WA, UK \\ ${ }^{2}$ BP Institute, University of Cambridge, Madingley Road, Cambridge CB3 0EZ, UK
}

(Received xx; revised xx; accepted xx)

The mixing properties of statically stable density interfaces subject to imposed vertical shear are studied using direct numerical simulations of stratified plane Couette flow. The simulations are designed to investigate possible self-maintaining mechanisms of sharp density interfaces motivated by Phillips' argument (Deep-Sea Res., vol. 19, 1972) by which layers and interfaces can spontaneously form due to vertical variations of diapycnal flux. At the start of each simulation, a sharp density interface with the same initial thickness is introduced at the midplane between two flat, horizontal walls counter-moving at velocities $\pm U_{w}$. Particular attention is paid to the effects of varying Prandtl number $\operatorname{Pr} \equiv \nu / \kappa$, where $\nu$ and $\kappa$ are the molecular kinematic viscosity and diffusivity respectively, over two orders of magnitude from $0.7,7$ to 70 . Varying $\operatorname{Pr}$ enables the system to access a considerable range of characteristic turbulent Péclet numbers $P e_{*} \equiv \mathcal{U}_{*} \mathcal{L}_{*} / \kappa$, where $\mathcal{U}_{\star}$ and $\mathcal{L}_{*}$ are characteristic velocity and length scales, respectively, of the motion which acts to 'scour' the density interface. The dynamics of the interface vary with the stability of the interface which is characterised by a bulk Richardson number $R i \equiv b_{0} h / U_{w}^{2}$, where $b_{0}$ is half the initial buoyancy difference across the interface and $h$ is the half height of the channel. Shear-induced turbulence occurs at small $R i$, whereas internal waves propagating on the interface dominate at large $R i$. For a highly stable (i.e. large $R i$ ) interface at sufficiently large $P e_{*}$, the complex interfacial dynamics allow the interface to remain sharp. This 'selfsharpening' is due to the combined effects of the 'scouring' induced by the turbulence external to the interface and comparatively weak molecular diffusion across the core region of the interface. The effective diapycnal diffusivity and irreversible buoyancy flux are quantified in the tracer-based reference coordinate proposed by Winters \& D'Asaro ( $J$. Fluid Mech., vol. 317, 1996) and Nakamura (J. Atmos. Sci., vol. 53, 1996), which enables a detailed investigation of the self-sharpening process by analysing the local budget of buoyancy gradient in the reference coordinate. We further discuss the dependence of the effective diffusivity and overall mixing efficiency on the characteristic parameters of the flow, such as the buoyancy Reynolds number and the local gradient Richardson number, and highlight the possible role of the molecular properties of fluids on diapycnal mixing.

\section{Key words:}




\section{Introduction}

In stably stratified flows in the ocean and atmosphere, it is not uncommon to observe step-like structures in the vertical profile of density with layers of nearly uniform density separated by sharp interfaces, see e.g. figure 10.1 of Turner (1973) showing a steplike temperature profile (although in this example the temperature changes can be at least compensated by changes in salinity). Other examples include the microstructure measurements by Gregg (1980) and those described in section 7.1 of Thorpe (2005). The flux-gradient paradigm proposed by Phillips (1972) is often used to explain the formation of such structures (while alternative mechanisms including internal wave straining have also been proposed, see e.g. Thorpe 2005, 2016). Phillips argued that the decrease of buoyancy flux with increasing buoyancy gradient leads to a vertical divergence of flux which then drives the spontaneous layering of buoyancy from an initially linear profile. Such a mechanism was also considered by Posmentier (1977), and the formation of steplike structures was observed in laboratory, e.g. by Ruddick et al. (1989). In this paper, we adopt a similar perspective to Phillips, in that we examine the vertical variation of diapycnal mixing properties such as diapycnal diffusivity and flux. However, we are interested here in the robustness rather than the formation of a 'sharp' interface from an initially uniformly stratified fluid. We focus on whether these mixing properties can lead to the maintenance and possible reinforcement of an existing sharp density interface. Our considerations are based on analysing direct numerical simulations (DNS) of stratified plane Couette flows with a sharp density interface which is introduced, as an initial condition, at the midplane between two flat, counter-moving horizontal walls. The stratified interface may then evolve in time subject to the constant shearing imposed by the walls. The properties of the diapycnal mixing occurring across the density interface not only could vary with external flow parameters, but also may exhibit strong spatial variation in the vertical $z$-direction. This $z$-dependent variation is the key focus of our investigation.

Central to Phillips' argument is the flux-gradient relation due to the assumed inherent properties of stratified turbulence. The review by Linden (1979) of numerous experiments supported the existence of such a regime where flux decreases with gradient, i.e. the 'right flank' of Phillips' curve (figure 1). Subsequently, various possible flux-gradient relations in the right-flank regime have been discussed, e.g. see figure 1 of Balmforth et al. (1998). Recently, statistical mechanics arguments developed by Venaille et al. (2017), assuming infinite Reynolds and Péclet numbers, suggest that some appropriate measure of the overall mixing efficiency, characterising the fraction of the kinetic energy loss by the fluid that leads an irreversibly gain in the potential energy due to mixing, varies non-monotonically with the overall gradient Richardson number if the background buoyancy profile contains a layered structure, whereas such a mixing efficiency asymptotes to a constant value of approximately 0.25 if the background buoyancy gradient is uniform. This suggests that the mixing properties of a sharp density interface may vary significantly from that of a linearly varying density profile (e.g. Shih et al. 2005). In this paper, we investigate the following four specific questions about the mixing properties of a density interface subject to imposed vertical shear.

(i) Does the diapycnal flux completely vanish when the stratification is particularly strong, or does the mixing efficiency saturate to a constant as in standard turbulence parameterizations (e.g. Mellor \& Yamada 1982), and as apparently observed in vertically stratified Taylor-Couette flow between two concentric cylinders by Oglethorpe et al. (2013)?

(ii) Does the molecular diffusivity of the fluid affect the overall mixing properties of 


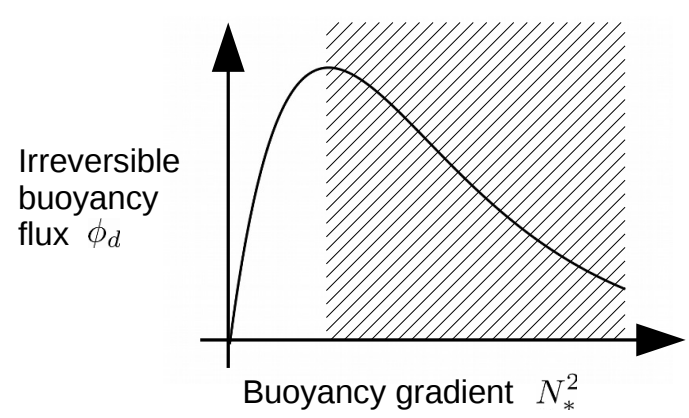

Figure 1: A schematic representation of the functional dependence of the irreversible buoyancy flux $\phi_{d}$ in terms of the buoyancy gradient $N_{\star}^{2}$, i.e. Phillip's flux-gradient curve. The definitions of $\phi_{d}$ and $N_{*}^{2}$ are discussed further in $\S 3$. The shaded portion corresponds to the regime in which the flux decreases with the gradient, i.e. the 'right flank' of the curve, and the unshaded portion corresponds to the 'left flank'. The asymptotic properties at sufficiently high buoyancy gradient are deliberately left open.

the system? In particular, how does the mixing efficiency in the layered system compare to recent numerical results obtained in other flow configurations, e.g. those studied by Salehipour et al. (2016b) and Maffioli et al. (2016)?

(iii) Does there exist a self-sustaining mechanism which can act to keep the interface sharp and maintain the layered structure?

(iv) If so, what are the ingredients of the mechanism, and is it possible to relate the self-sharpening process to vertical variations in the mixing properties, analogously to Phillips' argument?

It is well known that interfacial internal waves are important dynamical features associated with strongly stratified density interfaces. These waves may contribute, along with other relatively large-scale stirring processes, to the reversible component of buoyancy flux, thus introducing ambiguity to inferences of mixing from the conventional definition of buoyancy flux, i.e. the correlation between density and vertical velocity fluctuations (see e.g. the detailed discussion by Venayagamoorthy \& Koseff 2016). A rigorous framework concerning the potential energy balance in a control volume was developed by Winters et al. (1995) and employed for analysing the bulk properties (such as mixing efficiency) of mixing layers, e.g. by Caulfield \& Peltier (2000). A variant of the above formalism involves a tracer-based reference 'vertical' coordinate which was formulated by Winters \& D'Asaro (1996) and Nakamura (1996), which has been used, for example, to quantify mixing in idealised two-dimensional flows (Nakamura 1996; Shuckburgh \& Haynes 2003) and in large-scale geophysical situations (Marshall et al. 2006). In this paper, we use the formulation introduced by Winters \& D'Asaro (1996) and Nakamura (1996) to examine the structural details of fluxes and diffusivities as they vary in the tracer-based coordinate, here employed to describe three-dimensional direct numerical simulation data. As will be shown, this approach provides a useful framework for analysing the irreversible mixing, as well as the sharpening, or maintenance of a density interface.

The rest of the paper is structured as follows. In $\S 2$ we describe the numerical simulations of the layered stratified plane Couette flows and present qualitative observations on the time evolution of an originally sharp density interface. In $\S 3$ the formalism which involves a tracer-based reference coordinate is reviewed, and we propose a possible self- 
sharpening mechanism by examining the local budget of buoyancy gradient in such reference coordinates. In $\S 4$ we focus on the dynamics of a highly stable density interface and discuss the proposed self-sharpening mechanism in the framework that is presented in $\S 3$ using direct numerical simulation data. In $\S 5$ the dependence of effective diffusivity and overall mixing efficiency on the characteristic parameters of the flow is discussed. In $\S 6$ we provide some concluding remarks.

\section{Numerical simulations}

\subsection{Simulation set-up}

Direct numerical simulations (DNS) of layered stratified plane Couette (LSPC) flows are considered in this paper, and these simulations follow closely those of Deusebio et al. (2015) and Zhou et al. (2017). A full description of the DNS algorithms is presented in Taylor (2008). In these simulations, we consider the velocity vector $\mathbf{u}=(u, v, w)$ in the coordinate system $\mathbf{x}=(x, y, z)$, where $x$ and $y$ are the periodic (horizontal) directions and $z$ the wall-normal (vertical) direction. The incompressible Navier-Stokes equations under the Boussinesq approximation, i.e.

$$
\begin{aligned}
\frac{\partial \mathbf{u}}{\partial t}+\mathbf{u} \cdot \nabla \mathbf{u} & =-\frac{\nabla p}{\rho_{0}}+\nu \nabla^{2} \mathbf{u}+b \mathbf{e}_{z}, \\
\frac{\partial b}{\partial t}+\mathbf{u} \cdot \nabla b & =\kappa \nabla^{2} b, \\
\nabla \cdot \mathbf{u} & =0,
\end{aligned}
$$

are solved numerically, where $\nu$ and $\kappa$ are the kinematic viscosity and the scalar diffusivity respectively. The buoyancy

$$
b \equiv-\frac{\rho}{\rho_{0}} g
$$

is proportional to the gravity $g$ and the density deviation $\rho(\mathbf{x}, t)$ from the reference density $\rho_{0}$. Dirichlet boundary conditions for both velocity and buoyancy are applied at two horizontal non-slip walls as shown in figure 2 . The walls move at the same speed $U_{w}$ in opposite directions in $x$ with a fixed buoyancy difference of $2 b_{0}$ between them, i.e.

$$
(u, v, w, b)=\left( \pm U_{w}, 0,0, \pm b_{0}\right) \quad \text { at } \quad z= \pm h
$$

respectively, resulting in a statically stable stratified shear flow system. Note that we use the 'geophysical' coordinate system, where $z$ is the wall-normal vertical direction in which gravity acts, $x$ is the streamwise direction with the flow driven by the relative motion of the walls, and $y$ is the spanwise direction (see figure 2). Unless otherwise indicated in the remainder of the paper, velocities are normalised by $U_{w}$, lengths are normalised by $h$, buoyancy $b$ is normalised by $b_{0}$, and time $t$ is normalised by $h / U_{w}$ (i.e. the 'advective' time unit).

Three external parameters, i.e. the Reynolds number $R e$, the (bulk) Richardson number $R i$ and the Prandtl number $P r$, can be used to describe the flow. They are defined, respectively, as

$$
R e \equiv \frac{U_{w} h}{\nu}, \quad R i \equiv \frac{b_{0} h}{U_{w}^{2}} \quad \text { and } \quad \operatorname{Pr} \equiv \frac{\nu}{\kappa} .
$$

A total of 17 simulations are performed varying all three control parameters. The details of these simulations are summarised in table 1. Symbol types and colours (associated with each of the simulations) which are used in the subsequent figures are also shown in table 1. The choice of grid resolution in each simulation follows the specifications of Zhou 


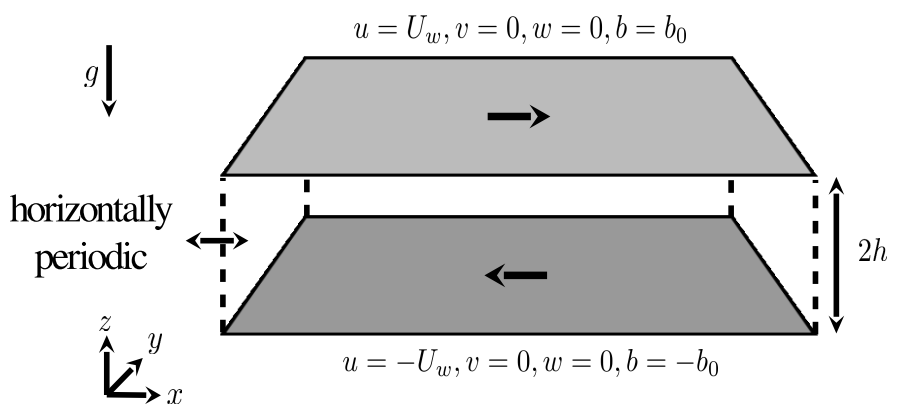

Figure 2: Configuration of stratified plane Couette flow and boundary conditions.

\begin{tabular}{|c|c|c|c|c|c|c|c|c|}
\hline Simulation & $R e$ & $\operatorname{Pr}$ & $R i$ & Symbol & Colour & $\left(N_{x}, N_{y}, N_{z}\right)$ & $\left(L_{x}, L_{y}, L_{z}\right)$ & Dyn. state \\
\hline 1 & 4250 & 0.7 & 0.01 & + & Red & $(256,256,129)$ & $(4 \pi, 2 \pi, 2)$ & T \\
\hline 2 & & & 0.02 & $\times$ & & & & $\mathrm{T}$ \\
\hline 3 & & & 0.04 & $\triangleright$ & & & & $\mathrm{T}$ \\
\hline 4 & & & 0.08 & $\triangle$ & & & & $\mathrm{L}$ \\
\hline 5 & & & 0.16 & 0 & & & & $\mathrm{~L}$ \\
\hline 6 & & & 0.32 & $\square$ & & & & L \\
\hline 7 & 4250 & 7 & 0.01 & + & Green & $(512,512,257)$ & $(4 \pi, 2 \pi, 2)$ & T \\
\hline 8 & & & 0.02 & $x$ & & & & $\mathrm{~T}$ \\
\hline 9 & & & 0.04 & $\triangleright$ & & & & $\mathrm{T}$ \\
\hline 10 & & & 0.08 & $\triangle$ & & & & $\mathrm{T}$ \\
\hline 11 & & & 0.16 & 0 & & & & L \\
\hline 12 & & & 0.32 & $\square$ & & & & L \\
\hline 13 & 4250 & 70 & 0.01 & + & Blue & $(768,768,769)$ & $(2 \pi, \pi, 2)$ & $\mathrm{T}$ \\
\hline 14 & & & 0.04 & $\triangleright$ & & & & $\mathrm{T}$ \\
\hline 15 & & & 0.08 & $\triangle$ & & & & $\mathrm{T}$ \\
\hline 16 & & & 0.32 & $\square$ & & & & $\mathrm{H}$ \\
\hline 17 & 14700 & 7 & 0.32 & $\square$ & Magenta & $(768,768,769)$ & $(2 \pi, \pi, 2)$ & L \\
\hline
\end{tabular}

Table 1: Summary of the numerical simulations of layered stratified plane Couette (LSPC) flows. $\left(N_{x}, N_{y}, N_{z}\right)$ are the number of grid points used in each direction, and $\left(L_{x}, L_{y}, L_{z}\right)$ are the lengths of computational domain. The last column lists the final dynamical state approached by each simulation: $T$ for 'turbulent'; $L$ for 'laminarising'; and $\mathrm{H}$ for 'Holmboe', all of which are described further in $\S 2.3$.

et al. (2017) for fully developed turbulent stratified plane Couette flows. The values of $\operatorname{Pr}$ considered in this paper include 0.7, 7 and 70. The first two values correspond to heat in air $(\operatorname{Pr}=0.7)$ and heat in water $(P r=7)$ respectively, and the largest value, i.e. 70 , is included in an attempt to investigate the poorly diffusive regime corresponding to salt in water with Schmidt numbers of approximately 700 (which is currently prohibitively costly to simulate with available resources). 


\subsection{Initial conditions}

The simulations considered in this paper are designed to examine the time evolution of an initially sharp density interface subject to imposed vertical shear and buoyancy difference across the interface. We are specifically interested in how the interface interacts with pre-existing turbulent motions that are external to the interface, i.e. what we will later describe as the 'scouring' mechanism for mixing (see Woods et al. 2010). The initial conditions used in our simulations are, therefore, considerably different from typical initial value problems concerning stratified shear instabilities investigated by run-down simulations. The latter simulations are typically initialised by specific mean profiles of $u(z)$ and $b(z)$ within a 'clean' laminar background with turbulence generated only by the break down of the instability itself, as in e.g. computational studies of Kelvin-Helmholtz and Holmboe instabilities (Salehipour \& Peltier 2015; Salehipour et al. 2016a).

The initial velocity field $\mathbf{u}(\mathbf{x}, t=0)$ for our 'production' simulations is obtained by auxiliary simulations performed in two stages: first, unstratified plane-Couette flow $(R i=$ 0 ) is simulated until it reaches a fully turbulent statistically stationary state. The purpose of this step is to produce a fully turbulent flow field spanning the channel gap. Second, in a 'relaxation stage' a sharp density interface with a hyperbolic tangent profile in $z$ :

$$
b(z)=b_{0} \tanh \left(\frac{z}{\delta_{0}}\right),
$$

where $\delta_{0} / h=0.08$, is introduced. The value of $\delta_{0} / h$ controls the initial 'sharpness' of the interface, i.e. the thickness of the interface, $\delta_{0}$, as compared to the half channel gap length, $h$, which characterises the length scale typical of large-scale energy-containing eddies in the turbulence between and wall and the density interface. Although it would be of interest to explore the dynamical effects of varying this ratio, for clarity we here only consider one specific value, sufficiently small so that the interface is adequately 'sharp'. All relaxation simulations are performed at $(R i, P r)=(0.08,0.7)$ and the Reynolds number is the same as in the unstratified simulation. The purpose of the relaxation stage is to reduce the excessive amount of turbulent kinetic energy (TKE) locally at the centre of the channel gap around the interface, so that the interface maintains its structural integrity at least at the beginning of the main 'production' simulations. This TKE reduction is achieved by resetting $\langle b\rangle(z)$, i.e. the mean value of $b$ averaged over a horizontal plane, to the initial hyperbolic tangent profile (2.5) at the end of every time step in the simulation, while allowing the perturbations $b^{\prime}(\mathbf{x}, t)=b(\mathbf{x}, t)-\langle b\rangle(z)$ and velocity field $\mathbf{u}(\mathbf{x}, t)$ to evolve in time. The strong stratification which is artificially maintained by resetting the mean buoyancy profile suppresses the turbulent motions in the vicinity of the interface and hence reduces the local values of TKE.

The volume-integrated TKE value reaches a minimum after running the relaxation procedure for $t \approx 60 h / U_{w}$, and the velocity field $\mathbf{u}(\mathbf{x})$ at this minimum TKE state is used to initialise the production simulations. A 'fresh' density field $b(z)$ following (2.5) is also introduced at the beginning of the production simulations, when the values of $\operatorname{Pr}$ and $R i$ are reset to those defined in Table 1 of a particular simulation. Three sets of initial $\mathbf{u}$ fields are obtained using the same procedure (but varying $R e$ or domain size), each applied to simulations $1-12,13-16$ and 17 , i.e. for simulations within each of the three groups, the initial $\mathbf{u}$ fields are identical.

Figure 3 shows typical vertical profiles describing the initial conditions of the simulations. The sharp buoyancy interface located at $z=0$ is embedded within a sheared velocity profile. The mean vertical shear is stronger both at the centre of the channel gap where the density interface is located and in the viscous wall regions. As previously discussed, the initial $\mathbf{u}$ field is turbulent with the profile (as shown in figure 3(b)) of the 

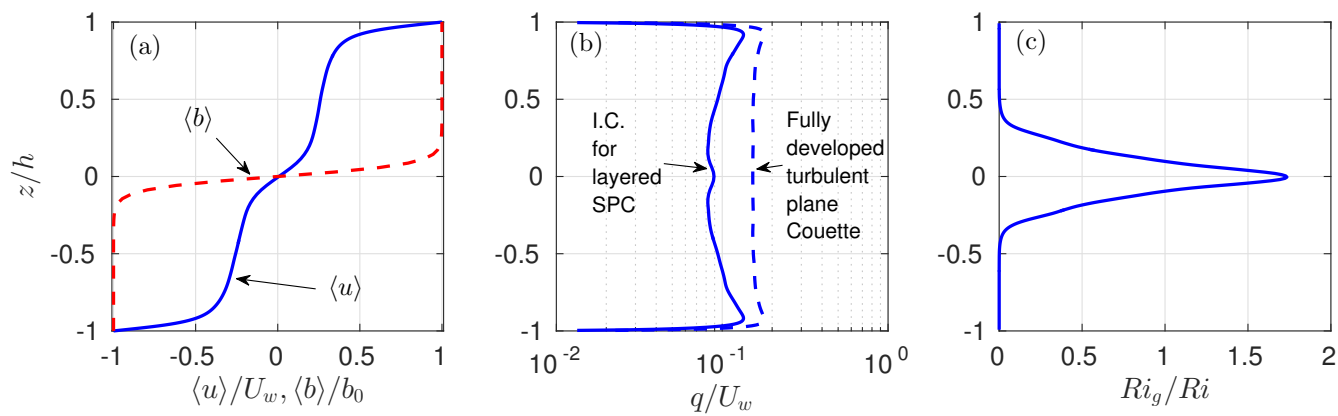

Figure 3: Vertical profiles of mean quantities corresponding to the initial condition used in the layered stratified plane Couette (LSPC) flow simulations with $R e=4250$. (a) Mean velocity $\langle u\rangle$ (plotted with a solid line) and buoyancy $\langle b\rangle$ (plotted with a dashed line). (b) Initial condition for the turbulent velocity scale $q$ for a layered stratified plane Couette flow simulation (plotted with a solid line) and a fully turbulent unstratified $(R i=0)$ plane Couette flow simulation at the same Re (plotted with a dashed line). (c) Profile of initial gradient Richardson number $R i_{g}(z, t=0)$, based on horizontal averages as defined in (2.7), divided by the bulk Richardson number $R i$.

turbulent velocity scale $q(z, t)$ defined as

$$
q(z, t) \equiv \sqrt{\left\langle u^{\prime 2}+v^{\prime 2}+w^{\prime 2}\right\rangle},
$$

where $\langle$.$\rangle indicates a spatial horizontal average over an x-y$ plane and $\left(u^{\prime}, v^{\prime}, w^{\prime}\right)$ denote fluctuation velocities from the horizontal mean. The magnitude of $q$ in the channel interior is approximately $10 \%$ of the wall speed $U_{w}$ and is reduced by approximately $40 \%$ from the unstratified fully turbulent plane Couette flow at the same Re. Again, this particular initial condition of $\mathbf{u}$ is designed specifically to prevent the interface from being broken up by strong turbulent motions when the interface is introduced at $t=0$. The mean gradient Richardson number,

$$
R i_{g}(z, t) \equiv \frac{N^{2}}{S^{2}}=\frac{\partial\langle b\rangle / \partial z}{(\partial\langle u\rangle / \partial z)^{2}},
$$

which is based on horizontal averages denoted by $\langle$.$\rangle , is plotted in figure 3(c) for t=0$. As expected, the $R i_{g}$ value peaks at the density interface centred at $z=0$ and is virtually zero within the uniform density layers above and below the interface, i.e. $|z / h| \gtrsim 0.4$.

\subsection{Qualitative observations}

Once initialised at $t=0$, the stratified interface is subject to the mean and turbulent motions maintained by the forcing of the walls. For flows with different external parameters, the interface exhibits different behaviours and approaches three possible dynamical states as tabulated in table 1 . The three possible states shown in figure 4 are:

(i) The 'turbulent' state $\mathrm{T}$ as shown in figure 4(a) for simulation 10. For relatively weakly stratified flows with $R i \leqslant 0.04$ for $\operatorname{Pr}=0.7$ or $R i \leqslant 0.08$ for $\operatorname{Pr}=7$ and 70 (see table 1), the stratification is too weak to suppress the turbulence. The interface soon becomes highly disordered with spatially intermittent shear-induced local overturns where vigorous mixing occurs. As a result, the sharpness of the interface is not robust, with the thickness of the interface increasing with time and the system approaching a fully turbulent, stratified, yet not definitely not layered state.

(ii) The 'Holmboe' state $\mathrm{H}$ is shown in figure 4(b) where the interface stays robust. The 
(a)

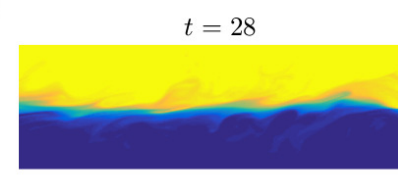

$t=167$
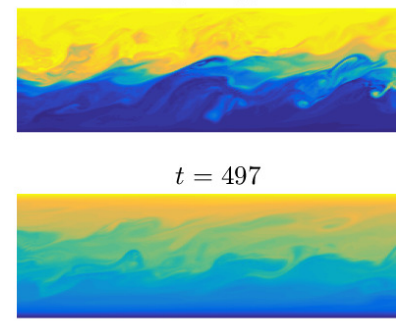

\begin{tabular}{lll}
\hline & 0 & 1
\end{tabular} (b)

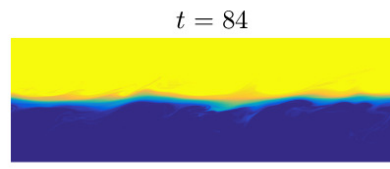

$t=165$

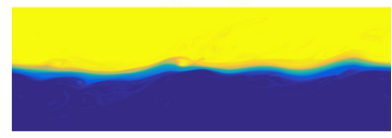

$t=348$

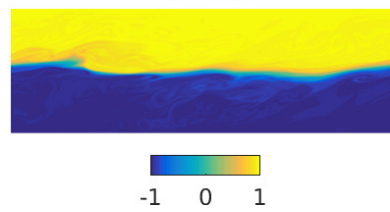

(c)

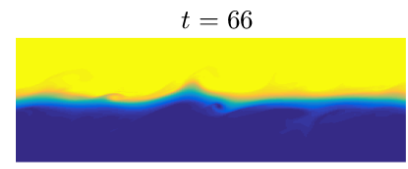

$t=140$

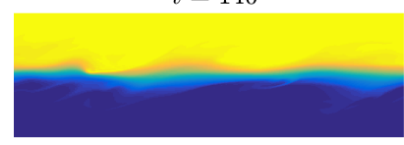

$t=365$

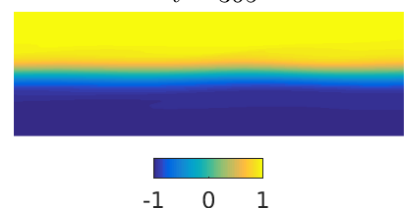

Figure 4: Side view of typical buoyancy field $b(x, z)$ at various times for (a) simulation 10: $(\operatorname{Pr}, R i)=(7,0.08)$, corresponding to T state, $(\mathrm{b})$ simulation 16: $(\operatorname{Pr}, R i)=(70,0.32)$, corresponding to $\mathrm{H}$ state, and (c) simulation 12: $(\operatorname{Pr}, R i)=(7,0.32)$, corresponding to $\mathrm{L}$ state. The visualisation window is $2 \pi h$ long in $x$ (corresponding to half of the domain length, $0.5 L_{x}$, for simulations 10 and 12 , and the full domain length, $L_{x}$, for simulation $16)$ and $2 h$ tall in $z$.

$\mathrm{H}$ state is observed in simulation 16 with large values of both $R i$ and $P r$, i.e. $R i=0.32$ and $\operatorname{Pr}=70$. Structures strongly reminiscent of 'Holmboe waves' (see e.g. figure 4 of Smyth et al. (1988) and figure 4 of Salehipour et al. (2016a)) appear to develop on the interface, and these structures prove to be long-lived and robust. 'Cusp' structures at the crests of the wave, along with concentrated spanwise vorticity, i.e. $\omega_{y}$, appear on both sides of the interface associated with these Holmboe-wave-like structures. As is typical, the cusps above and below the interface are observed to propagate in opposite directions. The vortices on either side of the interface act to entrain fluid from the interface, contributing to the 'wisps' structure in the lee of the 'cusps' in their direction of propagation, similar to the simulations of Smyth et al. (1988) and Salehipour et al. (2016a). It is important to note that all the propagating disturbances observed on the interface have characteristic phase speeds in the range $-U_{w}<c_{p h}<U_{w}$, and so none of the wave-like motions observed on the interface should be interpreted as 'pure' interfacial internal waves, unrelated to flow instabilities (specifically the Holmboe wave instability). The interface is observed to stay sharp, and the dynamics is dominated by internal waves rather than shearinduced turbulent overturns. The dynamics of the $\mathrm{H}$ state are also strongly reminiscent of the experimental observations of Holmboe waves on a sheared density interface by Strang \& Fernando (2001), who also reported buoyancy fluxes and entrainment rates based on planar laser-induced fluorescence measurements. The three-dimensional velocity and buoyancy fields obtained from direct numerical simulations allow us to consider the irreversible diapycnal mixing processes in detail, as is presented in the remainder of this paper.

(iii) The 'laminarising' state $\mathrm{L}$ is shown in figure $4(\mathrm{c})$ for simulation 12 . This $\mathrm{L}$ state exists at large $R i$ values for which stratification is able to suppress turbulence. Simulation 12 , shown as an example of the $\mathrm{L}$ state, has the same $R e$ and $R i$ values as simulation 16 , shown for the $\mathrm{H}$ state, but the $\operatorname{Pr}$ value is 7 instead of 70 . Internal waves similar to those in the $\mathrm{H}$ state appear at early times of the $\mathrm{L}$ state. The amplitude of the wave motion, 
(a)
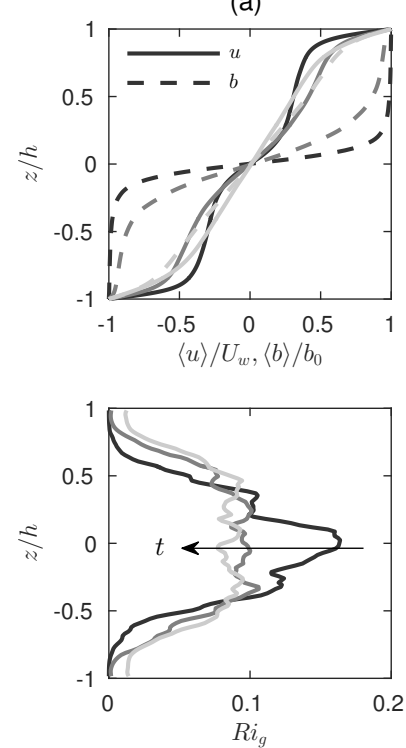

(b)
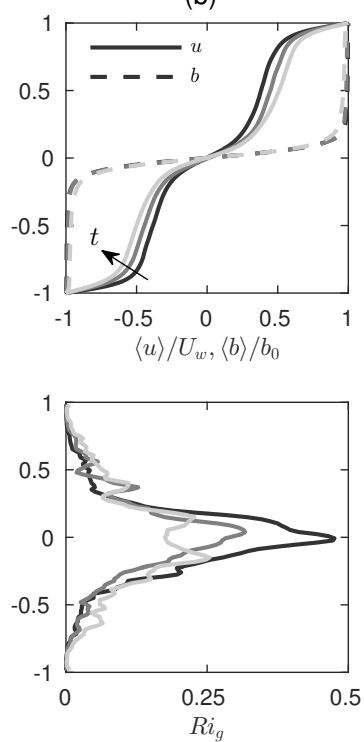

(c)
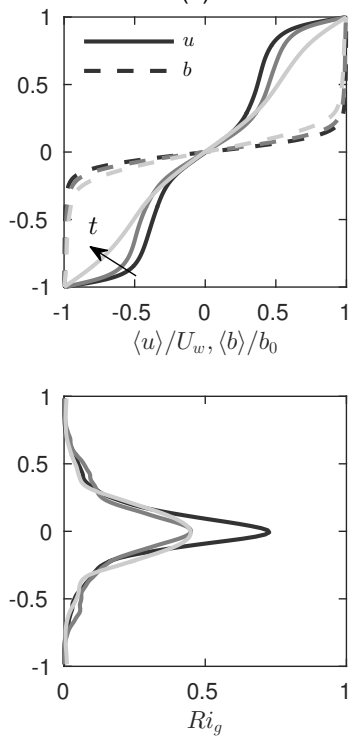

Figure 5: Horizontally-averaged velocity, buoyancy and gradient Richardson number profiles for: (a) simulation 10 at $(\operatorname{Pr}, R i)=(7,0.08)$ ( $\mathrm{T}$ state); (b) simulation 16 at $(\operatorname{Pr}, R i)=(70,0.32)$ (H state); and (c) simulation 12 at $(P r, R i)=(7,0.32)$ (L state). The profiles are sampled at the same times at which the buoyancy field is shown in figure 4 with lighter line shades corresponding to later times in each simulation.
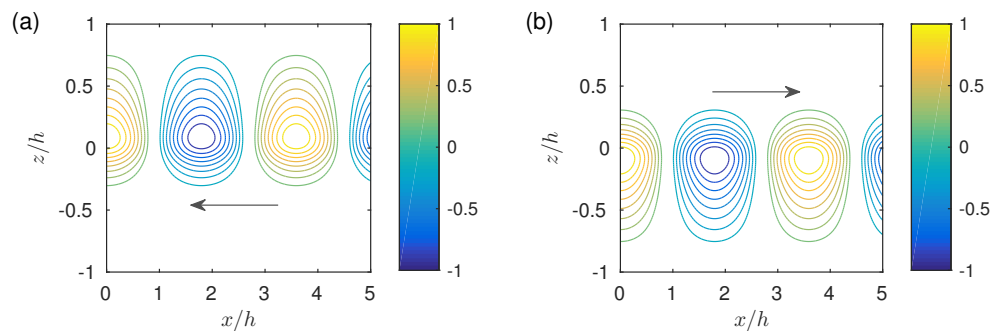

Figure 6: Typical structure of the vertical velocity eigenfunctions associated with the fastest growing modes of linear theory corresponding to Holmboe-type instabilities. The eigenfunctions are obtained for the mean profiles shown in figure 5 (b) at $t=84$ (darkest line) for simulation 16 ( $\mathrm{H}$ state). The eigenfunctions shown in both panels, (a) \& (b), have the same growth rate $\sigma \simeq 0.00171$ and equal and opposite real phase velocity $c_{p h} \simeq \mp 0.338$ (the arrow in each panel indicates the direction of $c_{p h}$ ). The streamwise wavenumber associated with these fastest growing modes is $k_{x} \simeq 1.75$.

however, noticeably decays with time, while the thickness of the interface gradually increases, presumably due to molecular diffusion. The flow is observed to approach the laminar steady state solution with $u / U_{w}=b / b_{0}=z / h$ (Eliassen et al. 1953).

As an aside, we can investigate the linear stability properties of the flows described above by examining the horizontally-averaged, instantaneous velocity and buoyancy profiles shown in figure 5. Simulations presented in figure 5 and the times at which the mean profiles are sampled are identical to those shown in figure 4 . In order to examine 
the linear stability of these mean profiles, the viscous, diffusive and stratified eigenvalue problem, e.g. as described in equations (3.6)-(3.7) of Eaves \& Caulfield (2017), is solved numerically using the procedure described in Smyth et al. (2011). Mean profiles associated with the T-state simulation 10 are shown in figure 5(a). While the gradient Richardson number, $R i_{g}$ associated with these averaged profiles is smaller than 0.2 (shown in the lower panel), the mean profiles are found to be linearly stable. However, the flow stays turbulent (see figure $4(\mathrm{a})$ ) as it evolves from the already turbulent initial condition (see figure 3(b)) to reaching the fully developed turbulent state (see e.g. Zhou et al. (2017)).

For the $\mathrm{H}$-state simulation 16 shown in figure $5(\mathrm{~b})$ and the L-state simulation 12 shown in figure $5(\mathrm{c})$, the mean profiles analysed are all unstable to instabilities which can be identified as being of Holmboe-type. This identification can be made for several reasons. The $R i_{g}$ distribution has the peaked structure associated with Holmboe-type instabilities. Furthermore, the velocity structure has strong shear over a relatively sharp interface, dropping to weaker shear either side. Such a structure is entirely characteristic of Holmboe-type instabilities, which can be interpreted as arising due to the interaction of an internal wave localised at the density interface, and a Doppler-shifted vorticity or 'Rayleigh' wave localised at the edge of the shear layer (Caulfield 1994; Baines \& Mitsudera 1994; Carpenter et al. 2011). Finally, the eigenfunction corresponding to the fastest growing Holmboe-type mode is plotted in figure 6, showing the characteristic structure centred above and below the 'sharp' density interface, leading to the characteristic propagation of the disturbance relative to the density interface (see Carpenter et al. (2010) for further discussion of instability classification in stratified shear flows).

It also is important to note that the profiles at $t=348$ for simulation 16 ( $\mathrm{H}$ state) are unstable also to Kelvin-Helmholtz-type instabilities, centred on the density interface. However, the Holmboe-wave-like structures only survive in the $\mathrm{H}$ state, but not in the $\mathrm{L}$ state, even though the linear analysis predicts the mean profiles are unstable to Holmboe instability in both cases. This analysis suggests that linear stability analysis based on the mean profiles should be used with caution when predicting the evolution of these density interfaces, at least when the underlying base flows are initially turbulent and the mean profiles vary significantly in time. This is not entirely surprising, because the substantial temporal and spatial variation of the actual streamwise velocity and buoyancy profiles about the horizontally-averaged mean profiles precludes infinitesimal perturbations experiencing for any extended period of time the notional profiles in which those infinitesimal perturbations are predicted to be (linearly) unstable.

As discussed above, our goal is to describe the behaviour of a pre-existing density interface subject to vertical shear from the perspective of diapycnal mixing. We are particularly interested in any self-sustaining (and hence inherently nonlinear) mechanism which keeps the interface sharp, and the existence of the $\mathrm{H}$ state described above provides a dataset which can be analysed to identify and describe such mechanisms. In the following section $(\S 3)$, the mathematical formalism we employ to describe the diapycnal mixing is described, and in $\S 4$ we focus on investigating the $\mathrm{H}$ state by comparing it to the $\mathrm{L}$ state as both $\mathrm{L}$ and $\mathrm{H}$ can occur in large- $R i$ strongly stratified systems. All $\mathrm{T}, \mathrm{H}$ and $L$ states are included in the considerations of mixing properties discussed in $\S 5$.

\section{Mathematical formulation}

\subsection{Tracer-based coordinate, flux and diffusivity}

The formalism developed by Winters \& D'Asaro (1996) and Nakamura (1996) is used to quantify the diapycnal mixing of the stratifying agent, i.e. the dynamic scalar tracer 
within the flow. This framework considers the mixing of a conserved tracer in a 'sorted' reference coordinate $z_{*}$. The definition of this $z_{*}$ coordinate relates to the 'background' buoyancy profile which is obtained by sorting all fluid parcels adiabatically to reach the minimum possible potential energy of the system, i.e. the background potential energy (see e.g. Winters et al. 1995). In the present study, we approximate the background buoyancy profile (or the 'sorted' profile) $b\left(z_{*}, t\right)$ via the probability density function (pdf) method introduced by Tseng \& Ferziger (2001) which avoids the explicit sorting procedure but is formally equivalent in the limit as the 'bins' used in constructing the pdf become arbitrarily small.

Following the Winters-D'Asaro-Nakamura formalism, the diapycnal flux $\phi_{d}$ across a specific isopycnal (constant buoyancy $b$ ) surface corresponding to a particular reference position $z_{*}$ can be defined by a simple flux-gradient relation

$$
\phi_{d} \equiv-\kappa_{e} \frac{\partial b}{\partial z_{\star}},
$$

where $\kappa_{e}\left(z_{*}, t\right)$ is an effective diapycnal diffusivity and the gradient $\partial b / \partial z_{*}$ can be obtained from the background buoyancy profile $b\left(z_{*}, t\right)$. The flux $\phi_{d}$ can be determined exactly from the instantaneous (dynamic) scalar field $b(\mathbf{x}, t)$ via the following relation

$$
\phi_{d}=-\kappa \frac{\partial z_{*}}{\partial b}\left\langle|\nabla b|^{2}\right\rangle_{z_{*}}
$$

where $\langle.\rangle_{z_{*}}$ indicates averaging over the isoscalar surface corresponding to the reference position $z_{*}$, and $|\nabla b|^{2}$ is given by the gradients of $b$ in the physical space $\mathbf{x}$. By definition, $b$ increases monotonically with $z_{*}$, i.e. $\partial z_{*} / \partial b>0$, and the flux $\phi_{d}$ is negative definite (down-gradient). It follows from (3.1) and (3.2) that the effective diffusivity $\kappa_{e}$ can be estimated by

$$
\kappa_{e}=\kappa\left(\frac{\partial z_{*}}{\partial b}\right)^{2}\left\langle|\nabla b|^{2}\right\rangle_{z_{\star}},
$$

which yields a positive-definite value of $\kappa_{e}$. The geometrical interpretation of (3.3) is given by equation (12) of Winters \& D'Asaro (1996), i.e.

$$
\kappa_{e}=\kappa\left(\frac{A_{s}}{A}\right)^{2} \geqslant \kappa,
$$

where $A_{s}$ is the area of the isopycnal surface corresponding to buoyancy $b$ at a given reference position $z_{\star}$. A given value of $z_{*}$ corresponds to a set of points in the physical $\mathbf{x}=$ $(x, y, z)$ coordinates. This set of points in $\mathbf{x}$ form the isopycnal surface(s) corresponding to the buoyancy value at the reference position $z_{*}$ in the sorted profile, i.e. $b\left(z_{*}\right)$. It is important to appreciate that the isopycnal surface(s) may have a distorted shape which may not be simply connected. $A$ in (3.4) is the area of the isopycnal surface projected onto a flat horizontal plane, i.e. the area of the flat undistorted surface. The increase of $A_{s}$ above $A$ is due to the straining imposed by the flow on the scalar field, and the effective diffusivity $\kappa_{e}$ can thus be greatly enhanced from the molecular value $\kappa$ due to the factor $\left(A_{s} / A\right)^{2}$.

\subsection{Evolution of background buoyancy profile}

Winters \& D'Asaro (1996) and Nakamura (1996) showed that the advection-diffusion equation of any conserved tracer in an incompressible flow can be written exactly as a one-dimensional diffusion equation in the reference $z_{*}$ coordinate:

$$
\frac{\partial b}{\partial t}=-\frac{\partial \phi_{d}}{\partial z_{*}}=\frac{\partial}{\partial z_{*}}\left(\kappa_{e} \frac{\partial b}{\partial z_{*}}\right) .
$$


Taking the derivative of (3.5) with respect to $z_{*}$ yields an evolution equation for the buoyancy gradient in the reference coordinate $N_{*}^{2} \equiv \partial b / \partial z_{\star}$ :

$$
\frac{\partial N_{*}^{2}}{\partial t}=\underbrace{\frac{\partial^{2} \kappa_{e}}{\partial z_{*}^{2}} N_{*}^{2}}_{\text {Source } \mathbb{S}(t)}+\underbrace{2 \frac{\partial \kappa_{e}}{\partial z_{*}} \frac{\partial N_{*}^{2}}{\partial z_{*}}}_{\text {Advection } \mathbb{A}(t)}+\underbrace{\kappa_{e} \frac{\partial^{2} N_{*}^{2}}{\partial z_{*}^{2}}}_{\text {Diffusion } \mathbb{D}(t)}
$$

The first bracketed term $\mathbb{S}(t)$ on the right hand side of (3.6) corresponds to a source/sink term for $N_{*}^{2}$ depending on the sign of the prefactor $\partial^{2} \kappa_{e} / \partial z_{*}^{2}$, the curvature of $\kappa_{e}$. The second bracketed term $\mathbb{A}(t)$ corresponds to the advection of $N_{\star}^{2}$ with a 'velocity' of $-2 \partial \kappa_{e} / \partial z_{\star}$. The third bracketed term $\mathbb{D}(t)$ corresponds to the diffusion of $N_{*}^{2}$ with the effective diffusivity $\kappa_{e}$ in the $z_{*}$ coordinate. Note that (3.6) can alternatively be written as

$$
\frac{\partial N_{\star}^{2}}{\partial t}=\frac{\partial^{2} \kappa_{e}}{\partial z_{\star}^{2}} N_{\star}^{2}+\frac{\partial \kappa_{e}}{\partial z_{*}} \frac{\partial N_{\star}^{2}}{\partial z_{*}}+\frac{\partial}{\partial z_{\star}}\left(\kappa_{e} \frac{\partial N_{\star}^{2}}{\partial z_{*}}\right),
$$

where the third term on the right hand side corresponds to the divergence of the diffusive flux $\kappa_{e} \partial N_{*}^{2} / \partial z_{*}$ in $z_{*}$, but we adopt the subdivision of terms in (3.6) for the rest of the paper. As will be shown in the following section $(\S 4)$, the diagnostic framework described here yields a robust description of the dynamics of temporally evolving density interfaces.

\section{Dynamics of highly stable interfaces}

\subsection{Structure of diapycnal flux and effective diffusivity}

In this section, we focus on simulations with $R i=0.32$, the largest bulk Richardson number which we have considered, and investigate the dynamics of interfaces with such strong stratification that is stable to shear-induced overturns. Figure 7 shows the profiles of effective diffusivity $\kappa_{e}$ and diapycnal flux $\phi_{d}$ in the $z_{*}$ coordinate. Several times are shown for simulation 12 (L state) at $(\operatorname{Pr}, R i, R e)=(7,0.32,4250)$ and for simulation 16 (H state) at $(\operatorname{Pr}, R i, R e)=(70,0.32,4250)$. Times associated with the profiles also correspond to the flow snapshots shown in panels (c) and (b) of figure 4 respectively.

As shown in figure $7(\mathrm{a})$, the buoyancy gradient $N_{*}^{2}$ at the midplane of the interface at $z_{*}=0$ decreases with time, and the thickness of the interface grows. The effective diffusivity $\kappa_{e}$ takes the molecular value $\kappa$ within the density interface located near $z_{*}=0$, and as the interface grows thicker, $\kappa_{e}$ approaches $\kappa$ over a broader range of $z_{*}$. This broadening suggests that the isopycnal surfaces are flattening, i.e. $A_{s} \rightarrow A$ as in (3.4), and the system is laminarising. The diapycnal flux $\phi_{d}$ varies significantly in $z_{*}$, and the divergence of the flux drives the broadening of the interface.

As is shown in figure 7(b), by varying $\operatorname{Pr}$ alone from 7 to 70 , simulation 16 is in the $\mathrm{H}$ state rather than the $\mathrm{L}$ state. The gradient $N_{*}^{2}$ at the midplane is observed to increase (though weakly) with time and the interface thickness remains approximately unchanged, which is consistent with the observations in figure 4(b) that the interface is robust and long-lived. The ratio $\kappa_{e} / \nu$ now takes smaller values at the midplane as the lower bound determined by molecular diffusivity $\min \left(\kappa_{e} / \nu\right)=\kappa / \nu=1 / \operatorname{Pr}$ is smaller due to the larger $\operatorname{Pr}$, which allows for a wide range of $\kappa_{e} / \nu$ from slightly above $1 / \operatorname{Pr} \sim O(0.01)$ around the midplane to $O(1)$ away from the interface at $z_{*} / h \approx \pm 0.1$. The flux $\phi_{d}$ is close to constant with $z_{*}$, and in the absence of a significant divergence of the flux, the strong gradient at the interface is expected to stay constant in time and last indefinitely.

The profiles shown in figure 7 also allow us to consider the role of various terms on the right hand side of (3.6) which govern the time evolution of the buoyancy gradient $N_{\star}^{2}$. In both simulations considered in figure 7 , the source term $\mathbb{S}(t)$ is positive and acts to 

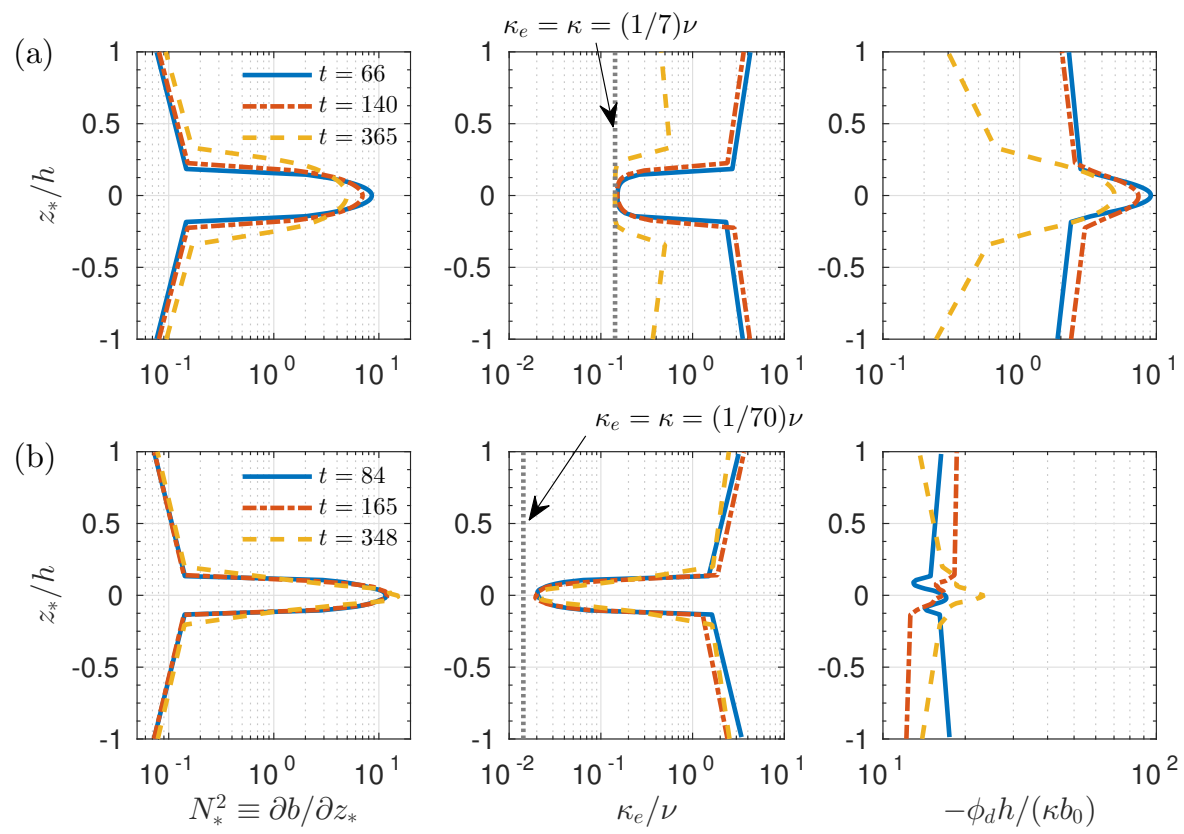

Figure 7: Profiles of: background buoyancy gradient $N_{*}^{2}$ (left column); effective diffusivity $\kappa_{e}$ normalised by molecular kinematic viscosity $\nu$ (middle column); and magnitude of diapycnal flux $\phi_{d}$ normalised by $\kappa b_{0} / h$ (right column). Upper row (a) corresponds to simulation 12 with $\mathrm{L}$ state at $(\operatorname{Pr}, R i)=(7,0.32)$, and lower row (b) corresponds to simulation 16 with $\mathrm{H}$ state at $(P r, R i)=(70,0.32)$. Both simulations are at $R e=4250$. Dotted vertical lines in the middle column correspond to the minimum possible value of $\kappa_{e}=\kappa$, or equivalently, $\kappa_{e} / \nu=1 / \operatorname{Pr}$. Profiles at various times are shown, and flow snapshots at these times can be found in figure 4 . Note that the horizontal axes are shown on different scales in the two subplots in the right column showing the $-\phi_{d}$ profiles.

sharpen the local gradient, but the prefactor corresponding to the curvature of $\kappa_{e}$, i.e. $\partial^{2} \kappa_{e} / \partial z_{*}^{2}$, is significantly larger for the $\mathbf{H}$ state. The advection term $\mathbb{A}(t)$ is expected to be nonpositive as $\partial \kappa_{e} / \partial z_{*}$ and $\partial N_{*}^{2} / \partial z_{*}$ tend to take opposite signs for a given $z_{*}$, but at the midplane of the interface $\mathbb{A}(t)$ is expected to be zero as $\partial \kappa_{e} / \partial z_{*}=\partial N_{\star}^{2} / \partial z_{*}=0$ at $z_{*}=0$ due to the symmetry of the profiles about the midplane. The diffusion term $\mathbb{D}(t)$ is expected to weaken the gradient within the interface as $\kappa_{e}$ is positive definite. Therefore, in order for an interface to be maintained, the source term $\mathbb{S}(t)$ must be able to counterbalance the effects of the other two terms. We investigate this balance quantitatively in $\S 4.2$.

The sign of $\partial^{2} \kappa_{e} / \partial z_{\star}^{2}$ serves as a simple diagnostic quantity to examine if any sharpening process is present around a density interface. Turbulence and/or vortical structures induced by Holmboe waves, which are displaced from the interface, could conceivably act on either side of the interface to 'scour' the material away from the interface via the 'wisps' structures that are clearly visible in figure 4(b). (Such a behaviour appears at least qualitatively to be occurring in the run-down simulations susceptible to Holmboe wave instabilities described in Salehipour et al. (2016a).) In this case, an isopycnal surface away from the midplane $z_{*}=0$ would have a more convoluted shape and thus larger surface area $A_{s}$ and hence larger $\kappa_{e}$ following (3.4). On the other hand, in the middle of the interface the flow exhibits minimal wave disturbances or turbulence, and the isopycnal 
surface is nearly flat with $A_{s} \approx A$. Thus $\kappa_{e}$ is expected to increase away from the midplane of the interface, consistent with the observations in figure 7 . It is then possible to have a positive curvature of the $\kappa_{e}\left(z_{*}\right)$ profile, i.e. $\partial^{2} \kappa_{e} / \partial z_{*}^{2}>0$, in the presence of mixing associated with scouring. When the scouring effect is large enough to overcome diffusion, i.e. $|\mathbb{S}(t)|>|\mathbb{D}(t)|$, the flow may act to enhance the local gradient $N_{\star}^{2}$. The reverse is true when one considers mixing due to large overturns, e.g. due to Kelvin-Helmholtz instability (KHI). The isopycnal surface in the overturning case is expected to have the most convoluted surface with large $A_{s} / A$ ratio in the core region of the KHI finite amplitude 'billow' where the maximum $\kappa_{e}$ is attained. The magnitude of $\kappa_{e}$ decreases with the distance to the midplane $z_{*}=0$, which may lead to $\partial^{2} \kappa_{e} / \partial z_{*}^{2}<0$ and thus negative values of $\mathbb{S}(t)$. The $\mathbb{S}(t)$ term then reduces the local $N_{*}^{2}$ value in concert with the diffusion term $\mathbb{D}(t)$, both acting to destroy the density interface through overturning dynamics.

\subsection{Time evolution of the buoyancy gradient with respect to $z_{\star}$}

In this subsection, we further examine the time evolution of various budget terms in (3.6) for the local gradient $N_{*}^{2}$. First, the integral thickness $\delta_{*}$ of the density interface can be calculated from the buoyancy profile by

$$
\delta_{*} \equiv \frac{1}{2 b_{0} h}\left[\int_{-h}^{0}\left(-b_{0}-b\right) z_{*} d z_{*}+\int_{0}^{h}\left(b_{0}-b\right) z_{\star} d z_{*}\right],
$$

and the buoyancy difference across the interface $\Delta b$ can be calculated as

$$
\Delta b \equiv \frac{1}{2}\left[b\left(z_{*}=\delta_{*}\right)-b\left(z_{*}=-\delta_{*}\right)\right] .
$$

The volume (depth) averaged value of an arbitrary quantity $\mathcal{F}\left(z_{*}, t\right)$ over the density interface $-\delta_{*}<z_{*}<\delta_{*}$ is denoted with an overbar, and defined as

$$
\overline{\mathcal{F}}(t) \equiv \frac{\int_{-\delta_{*}}^{\delta_{*}} \mathcal{F}\left(z_{*}, t\right) d z_{*}}{2 \delta_{*}} .
$$

A set of 'local' scalings can then be applied to the density interface to form the following dimensionless variables:

$$
\hat{z}_{*} \equiv \frac{z_{*}}{\delta_{*}}, \quad \hat{b} \equiv \frac{b}{\Delta b}, \quad \hat{t} \equiv \frac{\kappa t}{\delta_{*}^{2}} \quad \text { and } \quad \hat{\kappa}_{e} \equiv \frac{\kappa_{e}}{\kappa} .
$$

The governing equation for the buoyancy gradient $N_{\star}^{2}$ given by (3.6) can be rewritten as

$$
\frac{\partial}{\partial \hat{t}}\left(\frac{\partial \hat{b}}{\partial \hat{z}_{*}}\right)=\underbrace{\frac{\partial^{2} \hat{\kappa}_{e}}{\partial \hat{z}_{*}^{2}}\left(\frac{\partial \hat{b}}{\partial \hat{z}_{*}}\right)}_{\text {Source } \hat{\mathbb{S}}(t)}+\underbrace{2 \frac{\partial \hat{\kappa}_{e}}{\partial \hat{z}_{*}} \frac{\partial^{2} \hat{b}}{\partial \hat{z}_{*}^{2}}}_{\text {Advection } \hat{\mathbb{A}}(t)}+\underbrace{\hat{\kappa}_{e} \frac{\partial^{3} \hat{b}}{\partial \hat{z}_{*}^{3}}}_{\text {Diffusion } \hat{\mathbb{D}}(t)},
$$

with analogously scaled source, advection and diffusion bracketed terms.

In order to examine the evolution of the buoyancy gradient governed by (4.5) it is necessary to evaluate the gradients with respect to the tracer-based coordinate $\hat{z}_{*}$ of the effective diffusivity $\hat{\kappa}_{e}$ and the buoyancy $\hat{b}$. However, the noise contained in the $\hat{z}_{*}$ profiles associated with sampling issues (as shown in figure 8) tends to get amplified if finite differences are taken repeatedly on the $\hat{z_{*}}$ profiles to obtain the $\partial^{2} / \partial \hat{z}_{*}^{2}$ and $\partial^{3} / \partial \hat{z}_{*}^{3}$ gradients associated with higher order derivatives. Instead, we obtain an estimate of these gradients by first fitting polynomial functions to the observed $\hat{\kappa}_{e}\left(\hat{z}_{*}\right)$ and $\hat{b}\left(\hat{z}_{*}\right)$ profiles using a nonlinear least-squares algorithm and then calculate the gradients based on these 

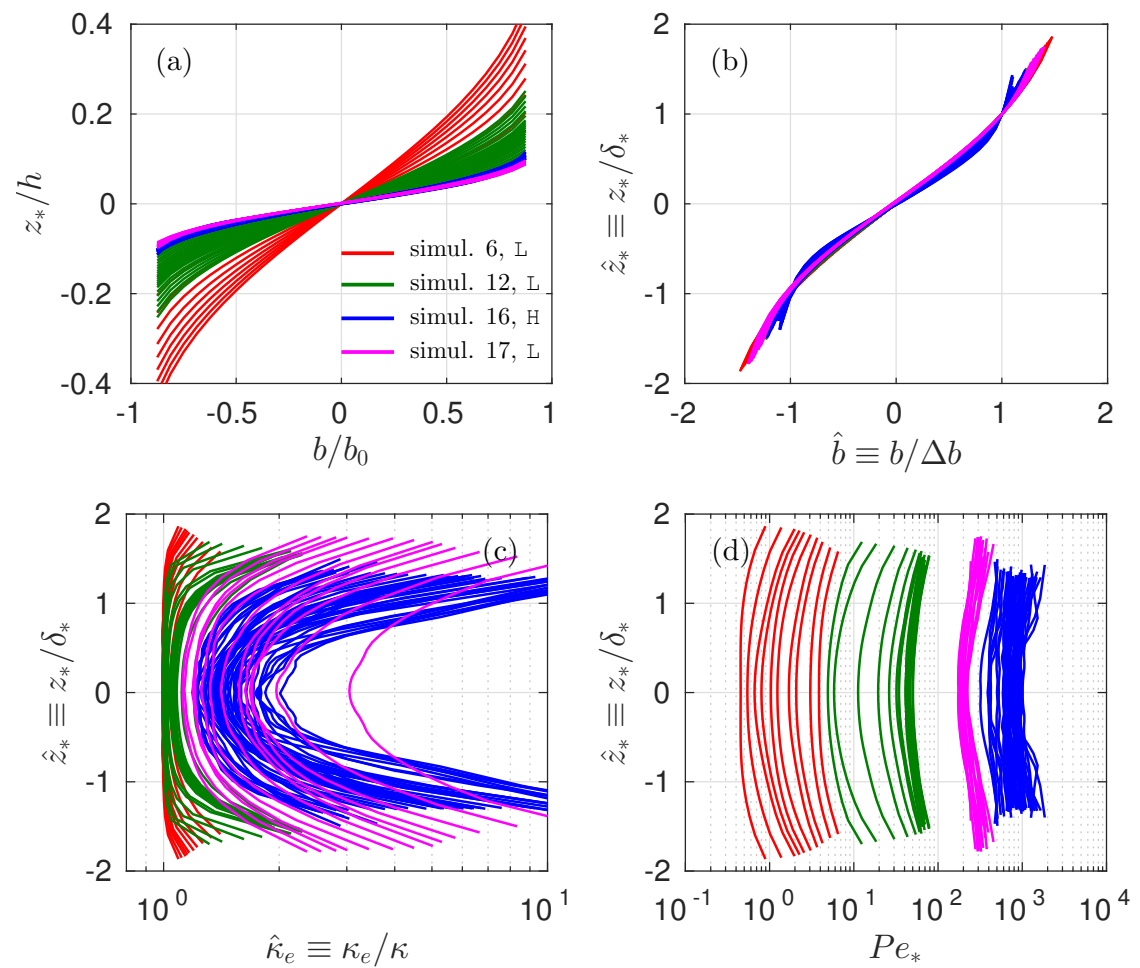

Figure 8: Sample profiles: of buoyancy (panels a \& b); effective diffusivity (panel c); and characteristic Péclet number $P e_{*}$, as defined in (4.6) (panel d). Multiple profiles are plotted for each simulation as the profiles evolve in time. Profiles are shown for: simulation 6 , an L state with $(\operatorname{Pr}, R e)=(0.7,4250)$ (plotted in red); simulation 12 , an $\mathrm{L}$ state with $(\operatorname{Pr}, R e)=(7,4250)$ (plotted in green); simulation 17 , an L state with $(\operatorname{Pr}, R e)=(7,14700)$ (plotted in magenta); and simulation 16 , an $\mathrm{H}$ state with $(\operatorname{Pr}, R e)=$ $(70,4250)$ (plotted in blue). In (b) the vertical extent of the buoyancy profile is rescaled by the interface thickness $\delta_{*}$ defined in (4.1) and its magnitude is rescaled by the buoyancy difference across the interface $\Delta b$ defined in (4.2).

fitted polynomial functions. Taking into account the symmetry of the profiles about the midplane $\hat{z}_{*}=0$, we assume that $\hat{\kappa}_{e}$ follows a parabolic profile $\hat{\kappa}_{e}=c_{1}+c_{2} \hat{z}_{*}^{2}$ and that $\hat{b}$ follows a cubic profile $\hat{b}=c_{3} \hat{z}_{*}+c_{4} \hat{z}_{*}^{3}$. It is worth noting that the rescaled buoyancy profiles $\hat{b}$ collapse reasonably well as shown in figure $8(\mathrm{~b})$.

The gradients of $\hat{b}$ with respect to $\hat{z}_{*}$ are $O(1)$ and they do not vary significantly from one simulation to another, as shown for example in figure 9 . On the other hand, the gradients of $\hat{\kappa}_{e}$ vary strongly between the various simulations. This can be seen in figure $8(\mathrm{c})$ where the rescaled $\hat{\kappa}_{e}\left(\hat{z}_{*}\right)$ profiles do not collapse. The curvature of the $\hat{\kappa}_{e}\left(\hat{z}_{*}\right)$ profile, i.e. $\partial^{2} \hat{\kappa}_{e} / \partial \hat{z}_{*}^{2}$, varies significantly between the various simulations and varies strongly in time, as is shown in figure $9(\mathrm{a})$.

In figures $10(\mathrm{a})$ and (b), the time evolution of the buoyancy gradient at the midplane $z_{*}=0$ is shown for the four simulations with $R i=0.32$. Except for simulation 16 which is in the $\mathrm{H}$ state, the gradient decreases with time for simulations 6,12 and 17, all of which are in the $\mathrm{L}$ state. In simulation 16 the density interface is maintained and the gradient at $z_{*}=0$ is weakly enhanced due to 'scouring' motions (see figure 4(b)). The time series 

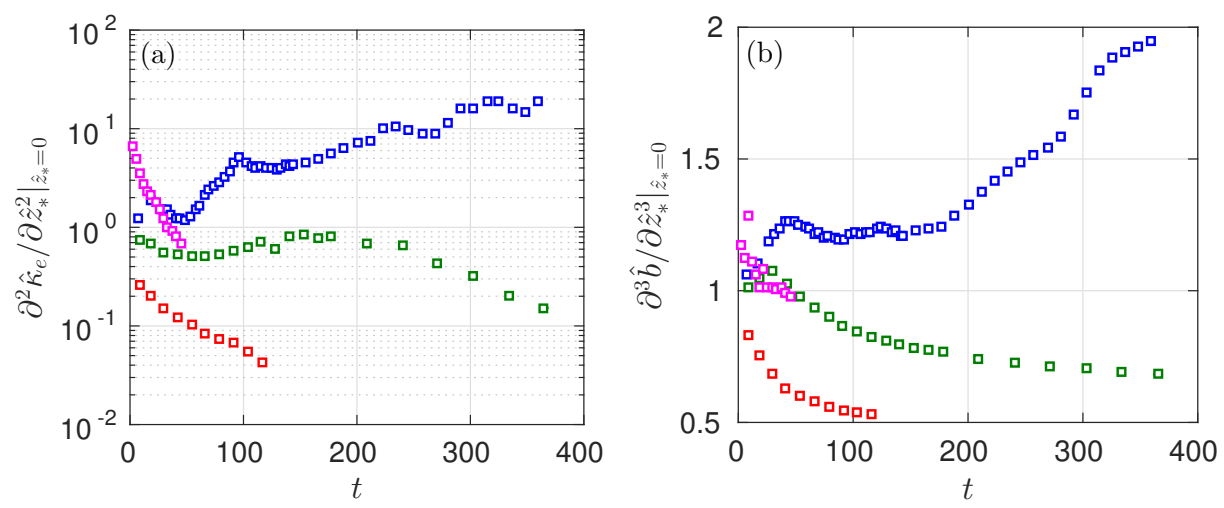

Figure 9: Time evolution of (a) $\partial^{2} \hat{\kappa}_{e} / \partial \hat{z}_{*}^{2}$ and (b) $\partial^{3} \hat{b} / \partial \hat{z}_{*}^{3}$ at the midplane of the interface $\hat{z}_{*}=0$. The colour conventions for various simulations are the same as those used in figure 8 .

of the source and diffusion terms in (4.5) which govern the time evolution of the local gradient $\partial \hat{b} / \partial \hat{z}_{*}$ are shown in figures $10(\mathrm{c})$ and (d). At the midplane of the interface, the advection term $\hat{\mathbb{A}}(t)$ is expected to be zero as both $\kappa_{e}$ and $\partial b / \partial z_{*}$ reach local extrema at $z_{*}=0$ due to symmetry (see figure 7 ). While for all simulations shown the source term $\hat{\mathbb{S}}(t)$ takes positive values, i.e. there is 'scouring' acting on the interface in all these cases, only in simulation 16 is this source term large enough to overcome the diffusion term $\hat{\mathbb{D}}(t)$, causing the local gradient $\partial \hat{b} / \partial \hat{z}_{*}$ to be enhanced. In the laminarising state cases, (simulations 6,12 and 17) however, the scouring effect is weak compared to the molecular diffusion which is characterised by the $\hat{\mathbb{D}}(t)$ term.

In figure 11 we examine the $\hat{z}_{*}$-dependence of the budget terms in (4.5) for a 'diffusing' interface in an $\mathrm{L}$ state simulation (simulation 12) for which the midplane gradient decreases (panel a) and a 'sharpening' interface in an $\mathrm{H}$ state simulation (simulation 16) for which the midplane gradient increases (panel b) respectively. In both cases, the advection term $\hat{\mathbb{A}}$ and the diffusion term $\hat{\mathbb{D}}$ both reduce the local gradient. In order for sharpening to occur, the source term $\hat{\mathbb{S}}$ has to outweigh $\hat{\mathbb{A}}$ and $\hat{\mathbb{D}}$, which is the case shown in panel (b). Note also that the enhancement of local gradients can only occur over a finite extent in $\hat{z}_{\star}$, i.e. sharpening around the centre of the interface comes at the expense of the buoyancy gradient immediately above and below the midplane at $\hat{z}_{\star}=0$.

\subsection{Effect of Péclet number and isopycnal displacement}

The terms $\partial \hat{b} / \partial \hat{z}_{*}, \hat{\kappa}_{e}$ and $\partial^{3} \hat{b} / \partial \hat{z}_{*}^{3}$ which appear in the source and diffusion terms in (4.5) are all of order unity at the midplane $z_{*}=0$, as can be seen in figures $8(\mathrm{c}), 9$ and $10(\mathrm{~b})$, respectively. Therefore, in order for $\hat{\mathbb{S}}$ to dominate $\hat{\mathbb{D}}$, the $\partial^{2} \hat{\kappa}_{e} / \partial \hat{z}_{*}^{2}$ term needs to be at least order unity or larger. In figure 12 , the values of $\partial^{2} \hat{\kappa}_{e} / \partial \hat{z}_{*}^{2}$ sampled at $z_{*}=0$ are plotted against the characteristic Péclet number of the flow. The characteristic Péclet number, which is a function of $z_{*}$ and $t$ is defined as

$$
P e_{*}\left(z_{*}, t\right) \equiv \frac{\mathcal{U}_{*}\left(z_{*}, t\right) \mathcal{L}_{*}\left(z_{*}, t\right)}{\kappa},
$$

where the characteristic turbulent velocity scale is defined as

$$
\mathcal{U}_{\star} \equiv \sqrt{\left\langle u^{\prime 2}+v^{\prime 2}+w^{\prime 2}\right\rangle_{z_{*}}},
$$



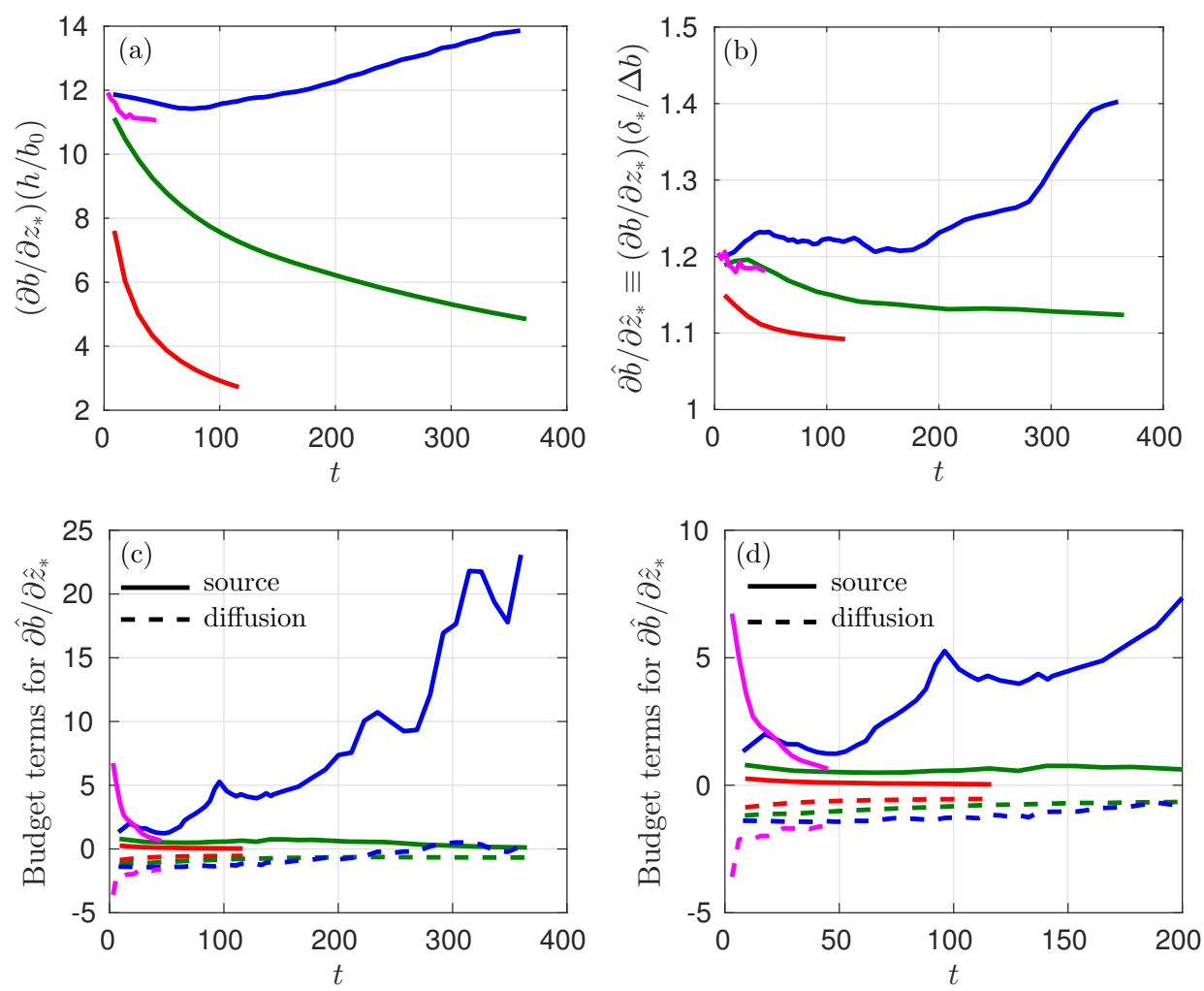

Figure 10: (a) and (b): Time evolution of the buoyancy gradient $N_{*}^{2} \equiv \partial b / \partial z_{*}$ at the midplane of the interface $z_{*}=0$. In (a) the gradient is scaled by $b_{0} / h$, and in (b) the local scaling $\Delta b / \delta_{*}$ is used. (c): Time evolution of the source term $\hat{\mathbb{S}}(t)$ solid lines) and the diffusion term $\hat{\mathbb{D}}(t)$ (dashed lines), as defined in (4.5), for $z_{*}=0$. (d): A zoomed view of panel (c) for $t<200$. Data are shown for: simulation 6 with $(\operatorname{Pr}, \operatorname{Re})=(0.7,4250)$ (plotted in red); simulation 12 with $(\operatorname{Pr}, R e)=(7,4250)$ (plotted in green); simulation 17 with $(\operatorname{Pr}, R e)=(7,14700)($ plotted in magenta $)$; and simulation 16 with $(\operatorname{Pr}, R e)=(70,4250)$ (plotted in blue), i.e. the same colour conventions as those used in figure 8 .

and the characteristic length scale is defined as

$$
\mathcal{L}_{*} \equiv \frac{\mathcal{U}_{*}}{\sqrt{\varepsilon_{*} / \nu}} .
$$

In the definition above, $\varepsilon_{*} \equiv\left\langle 2 \nu s_{i j} s_{i j}\right\rangle_{z_{*}}$ is the kinetic energy dissipation rate averaged for a given reference position $z_{*}$, and $s_{i j}$ is the rate of strain tensor associated with the full velocity field $\mathbf{u}$. The definition of the length scale $\mathcal{L}_{*}$ is analogous to the Taylor microscale which is often used to describe isotropic turbulence (see e.g. Pope 2000). The quantities $\mathcal{U}_{*}$ and $\mathcal{L}_{*}$ can be considered to be the characteristic velocity and length scales corresponding to the 'scouring' motion, and $P e_{*}$ measures the relative magnitude of scouring over molecular diffusion. $P e_{*}$ tends to increase weakly away from the midplane $z_{*}=0$ as shown in figure $8(\mathrm{~d})$.

As is plotted in figure 12 , the magnitude of $\partial^{2} \hat{\kappa}_{e} / \partial \hat{z}_{*}^{2}$ increases strongly with $\overline{P e}_{*}$, the depth-averaged Péclet number of a given profile, where the overline indicates an average as defined in (4.3). This figure illustrates the fact that $\hat{\kappa}_{e}$ profiles exhibit more 

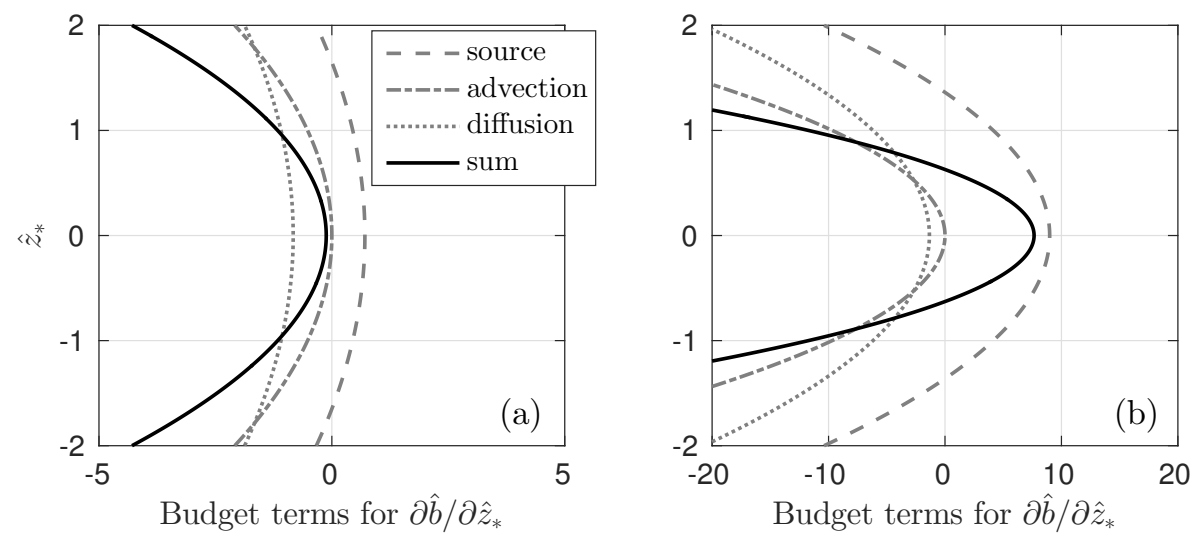

Figure 11: Variation with $\hat{z}_{*}$ of the various bracketed budget terms defined in (4.5) for: (a) a representative 'diffusing' interface in simulation 12 at $t \approx 100$; (b) a representative 'sharpening' interface in simulation 16 at $t \approx 200$.

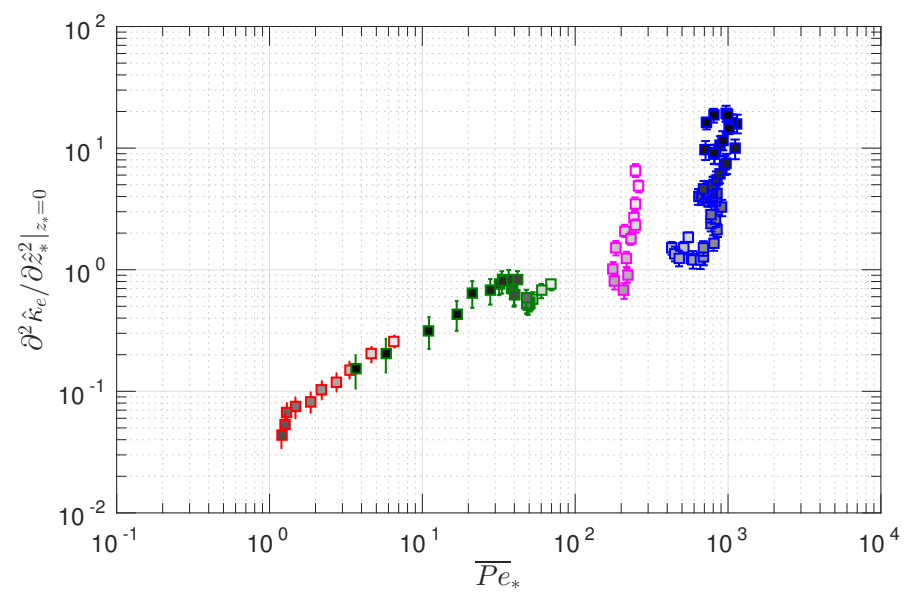

Figure 12: Variation of the curvature of the $\hat{\kappa}$ profile, i.e. $\partial^{2} \hat{\kappa}_{e} / \partial \hat{z}_{*}^{2}$, at the midplane of the interface $\hat{z}_{*}=0$, with the characteristic Péclet number $\overline{P e}_{*}$. The colour conventions for various simulations are the same as in figure 8. Darker filling colours of symbols correspond to later times in each simulation.

curvature as the effects of scouring become increasingly more important than molecular diffusion. Significantly, the curvature does not appear to vary systemically with other characteristic flow parameters such as buoyancy Reynolds number and local gradient Richardson number (as discussed in $§ 5$ ), the magnitude of which vary little across the four simulations shown in figure 12. The magnitude of $\partial^{2} \hat{\kappa}_{e} / \partial \hat{z}_{*}^{2}$ becomes larger than order unity for simulation 16 (plotted in blue) with $\overline{P e}_{*} \gtrsim 400$. As the flow evolves in this simulation (the filling colour of the symbol is darker and darker for later and later times), both $\overline{P e}_{*}$ and $\partial^{2} \hat{\kappa}_{e} / \partial \hat{z}_{*}^{2}$ increase with time. Other simulations with $\overline{P e}_{*} \lesssim 300$ do not have curvature $\partial^{2} \hat{\kappa}_{e} / \partial \hat{z}_{*}^{2}$ maintained at values larger than order unity. Although in simulation 17 (plotted in magenta) the $\partial^{2} \hat{\kappa}_{e} / \partial \hat{z}_{*}^{2}$ value starts with magnitude of order unity, it decays with time as the flow laminarises. It appears that there exists a transitional $\overline{P e}_{*}$ 


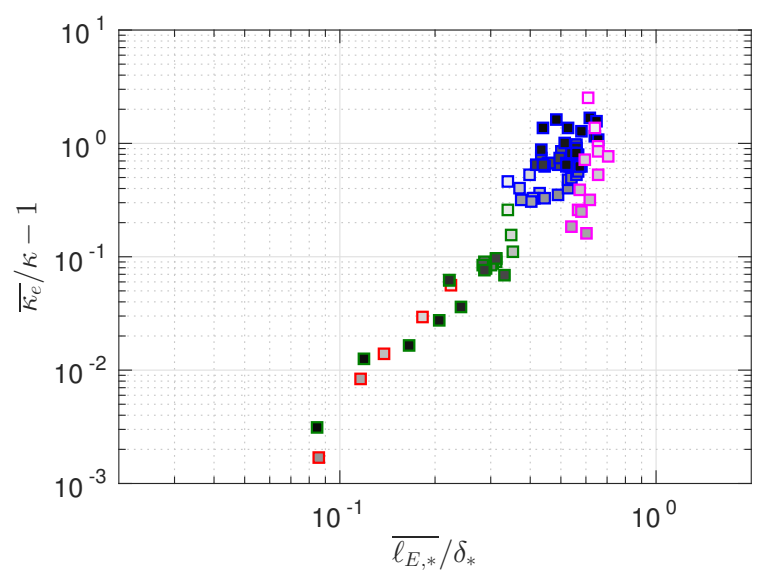

Figure 13: Variation of the depth-averaged enhancement ratio of diffusivity $\kappa_{e} / \kappa-1$ with the depth-averaged (across the interface) length scale ratio $\overline{\ell_{E, *}} / \delta_{*}$. The colour conventions for various simulations are the same as in figure 8. Darker filling colours of symbols correspond to later times in each simulation.

between 300 and 400 above which the scouring is able to overcome diffusion so that the curvature in $\hat{\kappa}_{e}$ can be maintained or enhanced.

Interestingly, this observation is reminiscent of the grid-stirred experiments (Crapper \& Linden 1974). In that paper, the behaviour of a density interface in the absence of mean shear is reported to vary significantly depending on whether an appropriate Péclet number is 'large' or 'small', i.e. whether the Péclet number based on the turbulent velocity and length scales at the interface is above or below about 200. For the highly stable, vertically sheared interfaces we examine here, the magnitude of the Péclet number appears to determine whether or not the scouring motion, which acts to sustain the interface, can overcome molecular diffusion, which acts to smooth the sharp gradient.

We also examine the weak enhancement of the effective diffusivity $\kappa_{e}$ relative to the molecular diffusivity $\kappa$ in the simulations of very stable interfaces. Figure 13 shows the depth-averaged enhancement ratio of effective diffusivity, $\overline{\kappa_{e}} / \kappa-1$, plotted against the ratio of the Ellison length scale to the integral thickness of the interface, $\overline{\ell_{E, *}} / \delta_{*}$, (a measure of the vertical isopycnal displacements) where the Ellison length scale is defined as

$$
\ell_{E, *}\left(z_{*}, t\right) \equiv \frac{\sqrt{\left\langle b^{\prime 2}\right\rangle_{z_{*}}}}{\partial\langle b\rangle_{z_{*}} / \partial z_{*}},
$$

and $b^{\prime} \equiv b-\langle b\rangle$ denotes the buoyancy fluctuation relative to the horizontal mean $\langle b\rangle$. Figure 13 suggests that the weak increase in $\kappa_{e}$ relative to $\kappa$ within the density interface is strongly correlated to the magnitude of isopycnal displacements. This observation reinforces the notion, which is encapsulated in (3.4), that diapycnal mixing is made more effective by a flow which creates larger isopycnal surface area for transport by molecular flux. In particular, enhancement of diffusion is achieved by the corrugation of isopycnal surfaces due to scouring motions acting on the very stable interfaces, an effect that is expected to be more significant as the isopycnal displacements increase in amplitude. 

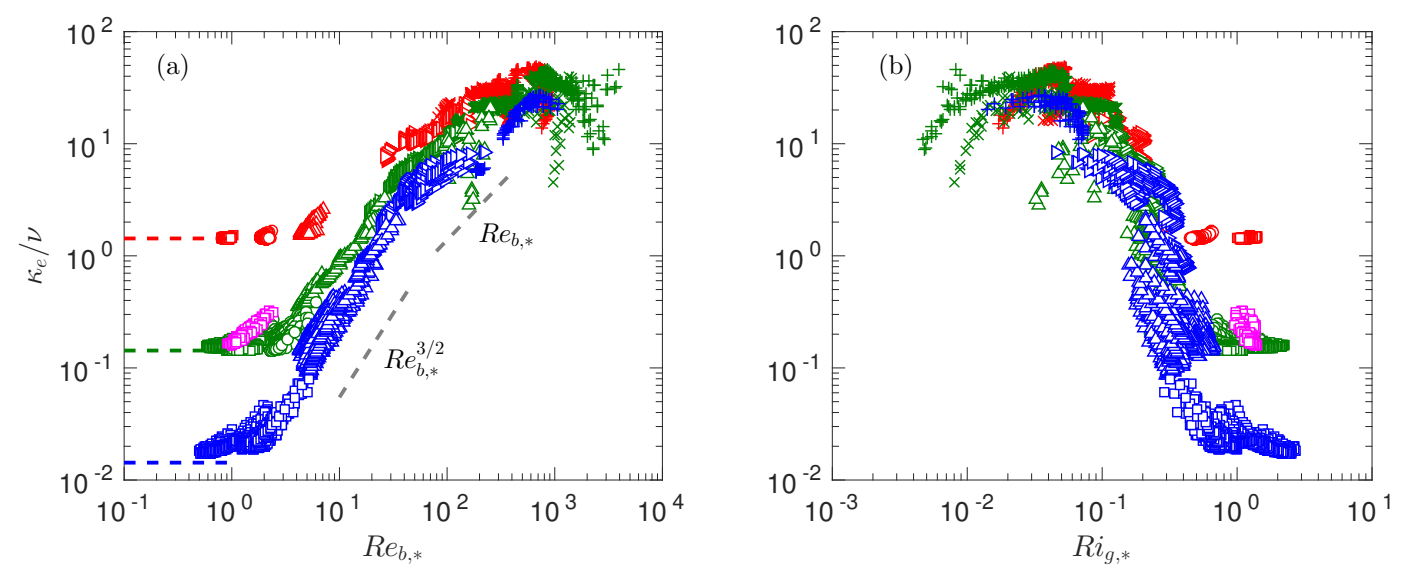

Figure 14: Variation of normalised $\kappa_{e}\left(z_{*}, t\right) / \nu$ with: (a) $R e_{b, *}\left(z_{*}, t\right)$; and (b) $R i_{g, *}\left(z_{*}, t\right)$. Horizontal dashed lines in (a) correspond to $\kappa_{e} / \nu=\kappa / \nu=1 / \operatorname{Pr}$ for $\operatorname{Pr}=0.7$ (red), 7 (green or magenta) and 70 (blue) respectively. Symbol conventions are listed in table 1.

\section{Mixing analysis in the tracer-based coordinate}

\subsection{Scaling of effective diffusivity}

In this section, we consider the variation of irreversible mixing properties with characteristic flow parameters in all three flow states, L, H and T. We start by investigating the effective diffusivity $\kappa_{e}$ as defined by (3.1). Following the Winters-D'Asaro-Nakamura formalism, $\kappa_{e}$ values are sampled locally at each $z_{*}$ using (3.3). All data points considered here are for $z_{*}$ locations sampled over the entire depth of the channel, i.e. $-h<z_{*}<h$ and for $t>10$ advective time units when the flow is observed to be free from initial transient effects due to the sudden introduction of the density interface at $t=0$. The values of $\kappa_{e}$, normalised by molecular kinematic viscosity $\nu$, are plotted against the locally sampled buoyancy Reynolds number $R e_{b, *}$ and gradient Richardson number $R i_{g, *}$, respectively, in figure 14. Specifically, $R e_{b, *}$ and $R i_{g, *}$ are defined in the tracer-based reference coordinate $z_{*}$ by

$$
R e_{b, *}\left(z_{*}, t\right) \equiv \frac{\varepsilon_{*}}{\nu N_{*}^{2}} \quad \text { and } \quad R i_{g, *}\left(z_{*}, t\right) \equiv \frac{N_{\star}^{2}}{S_{\star}^{2}},
$$

where $S_{\star} \equiv\langle\partial u / \partial z\rangle_{z_{*}}$ is the averaged vertical shear of streamwise velocity sampled over a given $z_{*}$ position.

Figure 14(a) indicates a clear dependence of $\kappa_{e} / \nu$ on both $R e_{b, *}$ and $\operatorname{Pr}$ at least for $R e_{b, *}<100$. For $R e_{b, *}=O(1)$ or smaller, $\kappa_{e}$ approaches the value $\kappa$, i.e. $\kappa_{e} / \nu \rightarrow 1 / \operatorname{Pr}$, in this 'molecular' regime (see e.g. Shih et al. 2005; Bouffard \& Boegman 2013). For $O(1)<R e_{b, *} \lesssim 30$, the scaling enters a 'buoyancy-controlled' regime where $\kappa_{e} / \nu \propto R e_{b, *}^{3 / 2}$ (c.f. Bouffard \& Boegman (2013) and the references therein). Consistent with Bouffard \& Boegman (2013), for a given $R e_{b, *}$ value, $\kappa_{e} / \nu$ decreases with increasing $\operatorname{Pr}$. For $30 \lesssim R e_{b, *} \lesssim 100$, i.e. the 'transitional' regime, $\kappa_{e} / \nu$ is proportional to $R e_{b, *}$, which agrees with the scaling of this regime described by Shih et al. (2005), although it is important to remember that the specific numerical values of the buoyancy Reynolds number depend on the choices for dissipation rate and buoyancy frequency made, which can of course vary between different analyses.

Within this 'transitional' regime, the weak dependence of $\kappa_{e} / \nu$ on $\operatorname{Pr}$ can still be 

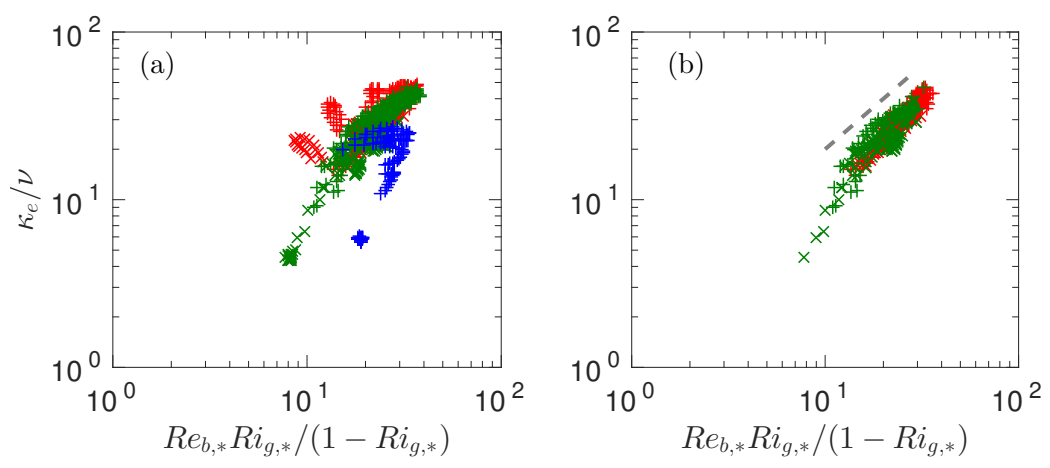

Figure 15: Application of the weakly stratified 'left-flank' scaling, i.e. $\kappa_{e} / \nu=R e_{b} R i_{g} /(1-$ $R i_{g}$ ), proposed for fully developed stratified plane Couette flow (Zhou et al. 2017), to the layered stratified plane Couette flow data. The 'left-flank' data points, with small bulk Richardson numbers $R i \leqslant 0.02$ are shown in (a) for $t>10$ and (b) for $t>60$. Dashed line in (b) indicates one-to-one slope. Symbol conventions are listed in table 1.

observed in our data. A simple power-law relation for $\kappa_{e} / \nu$ in terms of $R e_{b, *}$ is not identifiable for $\operatorname{Re}_{b, *} \gtrsim 100$ and the $\operatorname{Pr}$ dependence is also less distinct. Figure 14(b) shows the variation of $\kappa_{e} / \nu$ with $R i_{g, *}$ where the reverse trend in $R e_{b, *}$ can be observed, i.e. $\kappa_{e} / \nu$ in general decreases with increasing $R i_{g, *}$. This reversed trend is because, as will be shown in figure $18, R e_{b, *}$ and $R i_{g, *}$ are inversely correlated to each other in these simulations. The degree of scatter is greater in the $R i_{g, *}$ plot than in the $R e_{b, *}$ plot.

We now turn our attention to the $R e_{b, *} \gtrsim 100$ regime, where simple power laws in $R e_{b, *}$ do not appear to describe the data, as is shown in figure 14(a). These large $R e_{b, *}$ values are observed exclusively in the $T$ state where the flow remains turbulent despite the introduction of the density interface and approaches a fully-developed turbulent state (Zhou et al. 2017). In a fully turbulent stratified plane Couette flow, diapycnal mixing is characterised by a linear relation between the flux and gradient Richardson numbers, i.e. the turbulent Prandtl number $P r_{t} \equiv R i_{f} / R i_{g}$ is close to unity, where $R i_{f}$ is the flux Richardson number defined as the ratio of buoyancy flux and shear production. In other words, this is the typical behaviour on the weakly stratified 'left flank' of Phillips' fluxgradient curve (see figure 1). This results in a scaling of $\kappa_{e} / \nu=R e_{b} R i_{g} /\left(1-R i_{g}\right.$ ) (Zhou et al. 2017) which is tested in figure 15. In panel (a) some large deviations from this 'left flank' scaling can be observed, as the data points plotted include early-time points $(t<60)$ where the interface is undergoing shear-induced overturns. As the transition to stronger turbulence is close to completion at $t>60$, the $\kappa_{e} / \nu$ follows more closely the 'left-flank' scaling $R i_{f} \simeq R i_{g}$ for equilibrated weakly stratified shear flows, as shown for example in figure 13 of Deusebio et al. (2015).

\subsection{Scaling of volume-integrated mixing efficiency}

In this subsection, we consider the mixing efficiency of a density interface in the volumeintegrated sense. The framework of the analysis focusing on the available potential energy change in a control volume was proposed originally by Winters et al. (1995) and was employed subsequently to characterise the irreversible mixing efficiency in a given system by e.g. Caulfield \& Peltier (2000); Peltier \& Caulfield (2003). Here, we focus on the region within the density interface where a significant buoyancy gradient, $N_{*}^{2}$, is present and consider the integrated mixing properties over an interval in the $z_{*}$ coordinate with $-\delta_{*}<z_{*}<\delta_{*}$, where $\delta_{*}$ is the integral thickness of the interface in the $z_{*}$ coordinate as 
defined by (4.1). The integrated diapycnal flux is

$$
\Phi_{d}(t) \equiv-\bar{\phi}_{d} \cdot 2 \delta_{*}=\int_{-\delta_{*}}^{\delta_{*}}-\phi_{d}\left(z_{*}, t\right) d z_{*}=\int_{-\delta_{*}}^{\delta_{*}} \kappa_{e} \frac{\partial b}{\partial z_{*}} d z_{*},
$$

and the integrated dissipation is

$$
\mathcal{E}(t) \equiv \int_{-\delta_{*}}^{\delta_{*}} \varepsilon_{*}\left(z_{*}, t\right) d z_{*} .
$$

The overall irreversible mixing efficiency across the interface, which is defined as

$$
E_{t o t}(t) \equiv \frac{\Phi_{d}}{\Phi_{d}+\mathcal{E}},
$$

can then be estimated. In addition, it is possible to define a measure of mixing efficiency which excludes the laminar diffusion of the background profile with the laminar flux $\phi_{d, l a m} \equiv-\left(\partial b / \partial z_{*}\right) \kappa$, following the suggestion of Caulfield \& Peltier (2000) in an attempt to isolate the irreversible mixing inherently due to turbulent mixing processes. The corresponding integrated diapycnal flux can be estimated as

$$
\mathcal{M}(t) \equiv \int_{-\delta_{*}}^{\delta_{*}}-\left(\phi_{d}-\phi_{d, l a m}\right) d z_{*}=\int_{-\delta_{*}}^{\delta_{*}}\left(\kappa_{e}-\kappa\right) \frac{\partial b}{\partial z_{*}} d z_{*},
$$

and the corresponding 'turbulent' mixing efficiency can be estimated as

$$
E(t) \equiv \frac{\mathcal{M}}{\mathcal{M}+\mathcal{E}} .
$$

Figure 16 shows the total (turbulent and molecular) mixing efficiency $E_{\text {tot }}$ as a function of depth-averaged gradient Richardson number $\overline{R i}_{g, *}$ and buoyancy Reynolds number $\overline{R e}_{b, *}$, where the overbar indicates an average defined by (4.3). As shown in panel (a), $E_{t o t}$ increases with $\overline{R i}_{g, *}$ for $\overline{R i}_{g, *} \lesssim 0.1$ corresponding to the T state. The relation $E_{t o t}=R i_{g}$ plotted in a dashed line is equivalent to setting the turbulent Prandtl number $\operatorname{Pr}_{t}=1$, which appears to agree well with the data showing the typical 'left-flank' behaviour in Phillips' flux-gradient curve (figure 1). The data enter the 'right-flank' regime for $\overline{R i}_{g, *} \gtrsim 0.1$ where $E_{t o t}$ is observed to vary strongly with the molecular Prandtl number $\operatorname{Pr}$. Data points in this regime correspond mainly to the $\mathrm{L}$ and $\mathrm{H}$ states. Specifically, for $\operatorname{Pr}=0.7$ (plotted in red) $E_{t o t}$ continues to increase with $\overline{R i}_{g, *}$, because laminar diffusion, at least for these simulations, becomes important immediately after the flow enters the strongly stratified right flank. Non-monotonic behaviour of $E_{t o t}$ in $\overline{R i}_{g, *}$ is observed for $\operatorname{Pr}=7$ (plotted in green) and 70 (plotted in blue) where $E_{t o t}$ first decreases with $\overline{R i}_{g, *}$ and increases again when $\overline{R i}_{g, *}$ becomes sufficiently large due to the strength of the buoyancy gradient $\partial b / \partial z_{*}$. Shown also in figure 16(a) is the relation between $E_{t o t}$ and $\overline{R i}_{g, *}$ proposed by Venayagamoorthy \& Koseff (2016) plotted with a dashed-dotted line. While the relation is reasonably close to the data on the left flank, $E_{t o t}$ does not asymptote to a constant value of 0.25 as is predicted to occur in a linearly stratified system by Venaille et al. (2017), although as usual, it is important to remember that the definitions of mixing efficiency and Richardson number vary between analyses, and indeed the mechanisms by energy is injected into the flow also vary markedly.

When plotted against $\overline{R e}_{b, *}$, as is shown in figure $16(\mathrm{~b}), E_{\text {tot }}$ appears to collapse into single curves for each value of $\operatorname{Pr}$. For $R e_{b, *} \lesssim 100, E_{\text {tot }}$ takes larger values for smaller $\operatorname{Pr}$ at a given $R e_{b, *}$, and for $R e_{b, *} \gtrsim 100$, the dependence on $\operatorname{Pr}$ seems to disappear. Consistent with Shih et al. (2005), $E_{t o t}$ decreases with $\overline{R e}_{b, *}$ for $R e_{b, *} \gtrsim 100$. The Shih et al. (2005) data of $\operatorname{Pr}=0.72$ (plotted as grey squares) show consistency with the LSPC data for simulations with $\operatorname{Pr}=0.7$ (plotted in red) for $R e_{b, *}>O(1)$. 

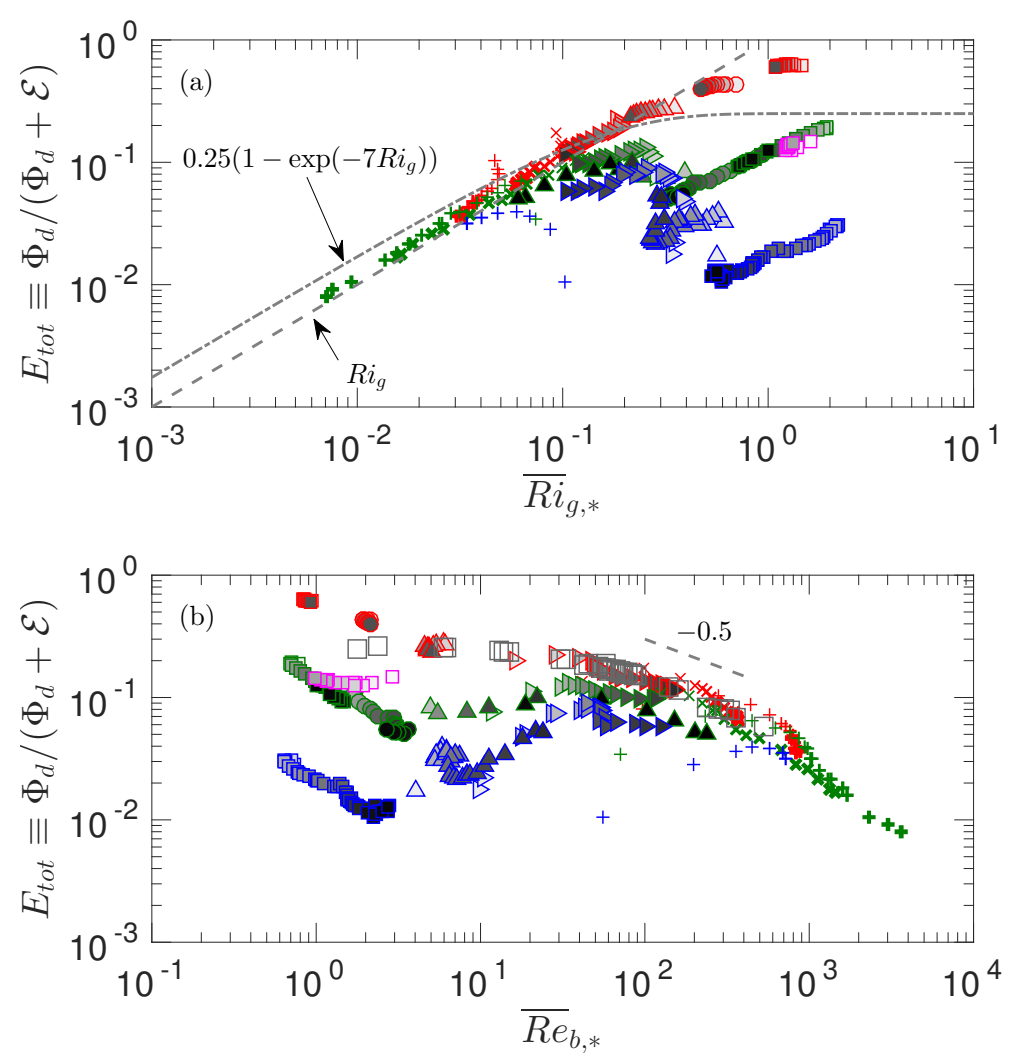

Figure 16: Variation of the time-dependent total mixing efficiency $E_{\text {tot }} \equiv \Phi_{d} /\left(\Phi_{d}+\mathcal{E}\right)$ across the density interface $-\delta_{*}<z_{*}<\delta_{*}$ with the corresponding depth-averaged: (a) $\overline{R i}_{g, *}$; and (b) $\overline{R e}_{b, *}$. Darker filling colours for the closed symbols and thicker lines for open symbols correspond to later times in each simulation. Symbol conventions are shown in table 1. Grey open squares in (b) correspond to data from Shih et al. (2005) with $\operatorname{Pr}=0.72$. In (a), a dashed line shows the relation $E_{t o t}=\overline{R i}_{g, *}$, and a dashed-dotted line shows the relation proposed by Venayagamoorthy \& Koseff $(2016), E_{t o t}=0.25[1-$ $\left.\exp \left(-7 \cdot \overline{R i}_{g, *}\right)\right]$.

Figure 17 shows the time-dependent 'turbulent' mixing efficiency $E$ as a function of $\overline{R i}_{g, *}$ and $\overline{R e}_{b, *}$. Interestingly, in panel (a) where $E$ is plotted against $\overline{R i}_{g, *}$, the strong dependence on $\operatorname{Pr}$ on the 'right flank' with $\overline{R i}_{g, *} \gtrsim 0.1$ vanishes when the laminar diffusion is excluded. As the flow further laminarises in the $L$ state, $E$ decreases with time (as shown by increasingly darker symbol fill colour). For the $\mathrm{H}$ state plotted in blue squares, however, the efficiency $E$ saturates to a value between $10^{-3}$ and $10^{-2}$. The same observation applies to the 'left-flank' in the $\overline{R e}_{b, *}$ plot shown in panel (b). The behaviour of $E$ follows closely that of $E_{t o t}$ shown in figure 16 for $\overline{R i}_{g, *} \lesssim 0.1$, as the contribution of laminar diffusion is negligible in flows where turbulent transport dominates, as expected. The data shown in panel (a) are also reminiscent of the results compiled by Fernando (1991) in his figure 16, although, again it is important to remember that the definitions of 'Richardson number' are different.

It is also important to appreciate the causes of the differences between the total mixing efficiency $E_{\text {tot }}$ (figure 16) and the turbulent mixing efficiency $E$ (figure 17). The definition 

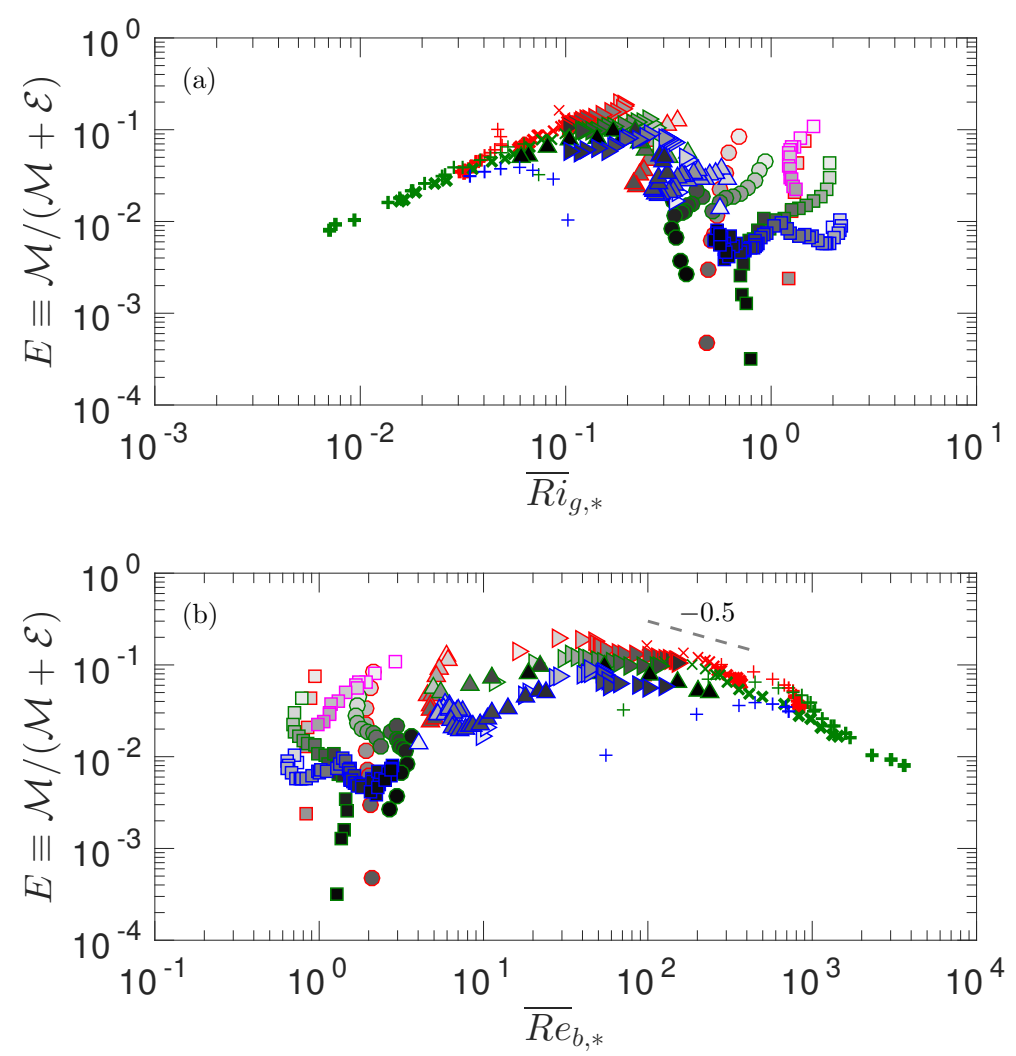

Figure 17: Variation of the time-dependent turbulent mixing efficiency $E \equiv \mathcal{M} /(\mathcal{M}+\mathcal{E})$ across the density interface $-\delta_{*}<z_{*}<\delta_{*}$ with the corresponding depth-averaged: (a) $\overline{R i}_{g, *}$; and (b) $\overline{R e}_{b, *}$. Darker filling colours for the closed symbols and thicker lines for open symbols correspond to later times in each simulation. Symbol conventions are shown in table 1 .

$E$ removing the purely diffusive component was proposed by Caulfield \& Peltier (2000) based on the assumption that the dominant mixing properties in flows unstable to KelvinHelmholtz instabilities (KHI) are associated with the breakdown of the primary KHI billows. By their very character, KHI billows are large-scale and dominated by inertial processes. As the Reynolds number of the flow increases, it is a reasonable hypothesis that the laminar 'mixing' dynamics will become increasingly insignificant. In the layered flow considered here, it is not at all clear that this assumption is valid, as even as the external $R e$ gets large, it is still expected that in the immediate vicinity of the density interface, diffusive 'laminar' dynamics will remain significant. This remaining significance is clearly implied by the spatial variation of $\kappa_{e}$ in strongly layered flows as shown in figure 7 .

\subsection{Comparison to mixing associated with Kelvin-Helmholtz instabilities}

In this section, we compare the mixing efficiency measured in our layered stratified plane Couette (LSPC) flows to the results obtained by simulating the turbulence induced by Kelvin-Helmholtz instabilities (KHI), a canonical flow configuration often employed to study mixing, e.g. by Caulfield \& Peltier (2000), Smyth et al. (2001), Mashayek et al. 

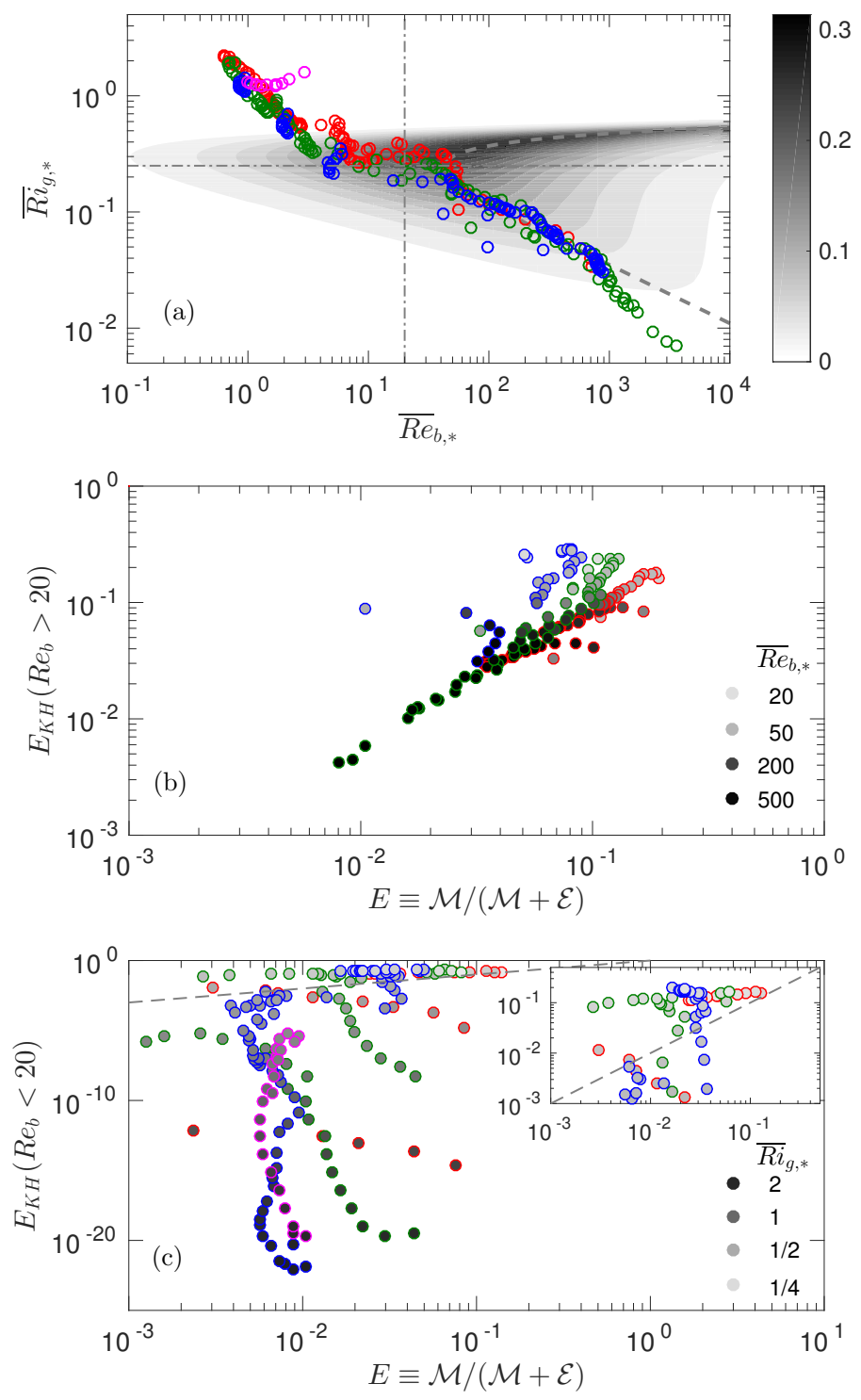

Figure 18: Comparison with the dual-parameter scaling for mixing efficiency $E \equiv \mathcal{M} /(\mathcal{M}+$ $\mathcal{E})$ in $\left(R i_{g}, R e_{b}\right)$ proposed by Salehipour et al. (2016b). In (a) the Salehipour et al. (2016b) predictions, denoted by $E_{K H}$, are plotted as contours, and the points in the parameter space accessed by LSPC simulations are plotted in circles where the colour conventions follow table 1 . The grey dashed line corresponds to where the maximum $E$ occurs for a given $R i_{g}$. The horizontal and vertical dashed-dotted lines correspond to $R e_{b}=20$ and $R i_{g}=1 / 4$ respectively. The predicted $E_{K H}$ values are plotted against the LSPC results in (b) and (c) for $R e_{b}>20$ and $R e_{b}<20$ respectively. Darker fill colour corresponds to larger values of $\overline{R e}_{b, *}$ in (b) and larger values of $\overline{R i}_{g, *}$ in (c). The dashed line in (c) and the insert plot corresponds to $E=E_{K H}$. 
(2013) and Salehipour \& Peltier (2015). Figure 18 compares our LSPC data to a recent study by Salehipour et al. (2016b) which attempted to parameterize $E$ as a function of appropriate measures of gradient Richardson number and buoyancy Reynolds number based on data from direct numerical simulation of KHI. As previously noted, it is very important to be cautious when comparing results from different analyses using different definitions of key parameters, and as described in detail in Salehipour \& Peltier (2015), the definitions of the gradient Richardson number and buoyancy Reynolds number used in Salehipour et al. (2016b) are somewhat different from those used here. To re-iterate, the $\overline{R i}_{g, *}$ and $\overline{R e}_{b, *}$ values for our LSPC data are first calculated 'locally' as a function of $z_{*}$ using the definitions given in (5.1), and are then averaged using the 'depth' integral (denoted with an overbar) as defined in (4.3). As can be seen in panel (a) of figure 18, $\overline{R i}_{g, *}$ and $\overline{R e}_{b, *}$ are strongly correlated to each other in the LSPC flows, i.e. $\overline{R e}_{b, *}$ tends to decrease with larger values of $\overline{R i}_{g, *}$. As a result, our data only access a subset of the parameter space. Interestingly, our data for $20 \lesssim R e_{b} \lesssim 1000$, which fall in the weakly stratified 'left flank' of Phillips curve, follow closely the trajectory of maximum $E$ for a given $\overline{R i}_{g, *}$ observed by Salehipour et al. (2016b). The LSPC data points do not access the most efficient regime observed by Salehipour et al. (2016b) when $\overline{R e}_{b, *} \gtrsim 20$ and $\overline{R i}_{g, *} \gtrsim 0.25$. For $\overline{R e}_{b, *} \gtrsim 20$, the LSPC data agree reasonably well with Salehipour et al. (2016b)'s prediction $E_{K H}$, as is shown in panel (b). The agreement, which seems to be improved for data points of larger $\overline{R e}_{b, *}$ values, is presumably due to the fact that the underlying flow dynamics is similar in LSPC and KHI simulations for these data points, i.e. shear-induced overturns dominate the diapycnal mixing in both cases. For the less energetic, more stratified data points with $\overline{R e}_{b, *} \lesssim 20$ (or $\overline{R i}_{g, *} \gtrsim 0.25$ ), there is poor agreement between $E_{K H}$ and $E$, as is shown in panel (c). The Salehipour et al. (2016b) scaling predicts larger efficiencies than those observed in the LSPC flow for small values of $\overline{R i}_{g, *} \lesssim 1 / 2$, as shown in the insert of panel (c). As $\overline{R i}_{g, *}$ increases further to $\overline{R i}_{g, *} \gtrsim 1 / 2$, $E_{K H}$ becomes virtually zero, whereas $E$ stays at small but significantly non-zero values. This weak but non-negligible mixing occurs in $\mathrm{L}$ and $\mathrm{H}$ states at the right flank of Phillips curve for which the diapycnal transport due to the scouring acting on a highly stable density interface plays a key role.

\subsection{Comparison to body-forced turbulence mixing}

Another highly relevant flow configuration in studying stratified turbulence is triply periodic forced turbulence simulations, e.g. by Brethouwer et al. (2007). Here we also compare our results with a recent study by Maffioli et al. (2016) who measured mixing efficiency from a series of body-forced stratified turbulence simulations (figure 19). Crucially, the flow in their study is energised by the use of body forcing in contrast to applying vertical shear driven at the boundaries in LSPC flow simulations, and only a statistically steady state is considered in Maffioli et al. (2016), whereas time-dependent mixing properties are captured in the LSPC flow data. Maffioli et al. (2016) observed the dependence of mixing efficiency on the turbulent Froude number $F r_{h} \equiv \varepsilon /\left(N \mathcal{U}^{2}\right)$, an equivalent of which can be estimated as $F r_{h, *}=\varepsilon_{*} /\left(N_{*} \mathcal{U}_{h, *}^{2}\right)$ in the $z_{*}$ coordinate, where $\mathcal{U}_{h, *} \equiv\left\langle u^{\prime 2}+v^{\prime 2}\right\rangle_{z_{*}}$ is the turbulent horizontal velocity scale, though once again caution must be applied when comparing specific numerical values of differently defined quantities. As shown in figure 19(a), plotting $E$ against the depth-averaged $\overline{F r}_{h, *}$ does not collapse the LSPC flow data completely, and the Maffioli et al. (2016) simulations have a significantly larger mixing efficiency. Furthermore, the LSPC flow never accesses the small Froude number regime identified by Maffioli et al. (2016), associated with an asymptotic (and constant) mixing efficiency. 

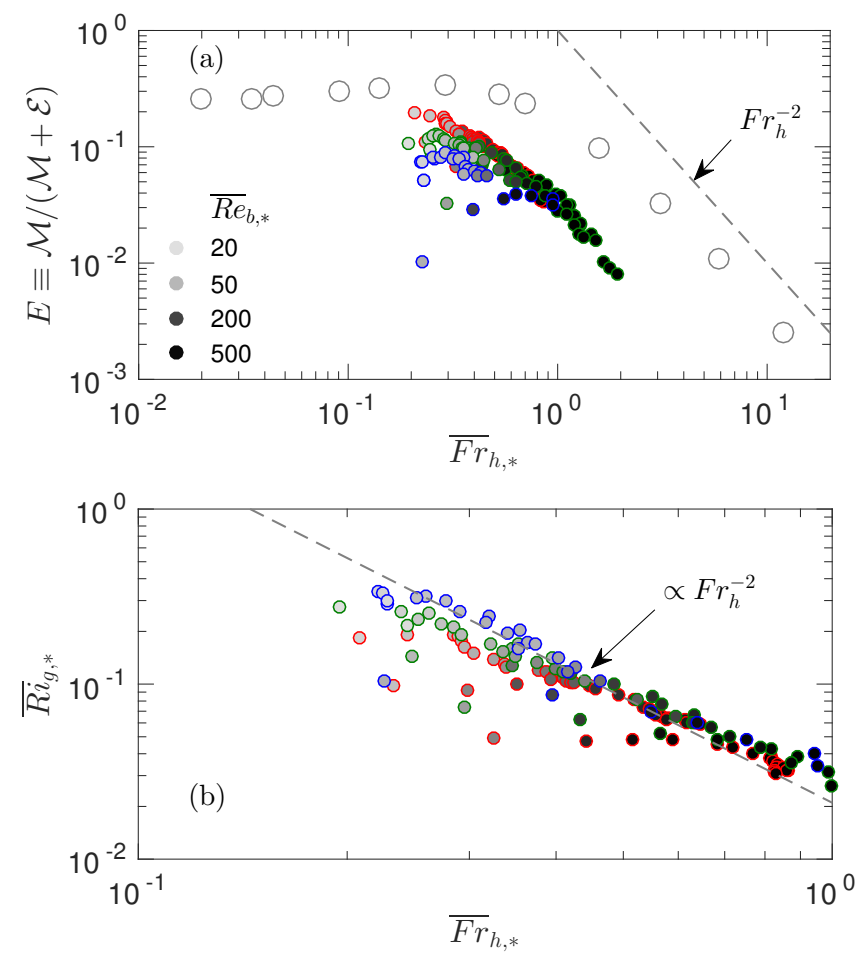

Figure 19: (a) Mixing efficiency $E \equiv \mathcal{M} /(\mathcal{M}+\mathcal{E})$ as a function of the depth-averaged horizontal Froude number $\overline{F r}_{h, *}$. The data from Maffioli et al. (2016) are plotted as grey circles. (b) $\overline{R i}_{g, *}$ as a function of $\overline{F r}_{h, *}$, where the dashed line corresponds to the $\mathrm{Ri}_{g} \propto \mathrm{Fr}_{h}^{-2}$ scaling for fully developed turbulent plane Couette flow (Zhou et al. 2017). Darker fill colour corresponds to larger values of $R e_{b}$ with samples shown in panel (a). Data points with $\overline{R e}_{b, *}>20$ are shown, consistent with the range investigated by Maffioli et al. (2016).

This difference appears to be related to the fundamental difference in the forcing, with the external wall-forcing always leading to weaker mixing. Interestingly, the $\mathrm{Fr}_{h}^{-2}$ scaling in the weakly stratified regime $\left(F r_{h}>1\right)$ of Maffioli et al. (2016) seems to apply also to the large- $\overline{R e}_{b, *}$ data points from LSPC flow, although the value of $E$ is roughly one order of magnitude smaller in LSPC flow for a given turbulent Froude number. Note that the scaling $E \propto F r_{h}^{-2}$ may be inherently connected to the scaling $E \propto R i_{g}$, because it can be shown in fully turbulent stratified plane Couette flow (Zhou et al. 2017) that $R i_{g} \propto \mathrm{Fr}_{h}^{-2}$, a relation which appears to hold, at least approximately, for the LSPC flow data shown in figure $19(\mathrm{~b})$.

\section{Concluding remarks}

We have examined irreversible diapycnal mixing quantified in the tracer-based coordinate $z_{*}$ following the Winters-D'Asaro-Nakamura formalism for layered stratified plane Couette flow simulations. The results presented include not only the bulk (volumeaveraged) properties of irreversible mixing, but also the structural details of effective diffusivity $\kappa_{e}$ and diapycnal flux $\phi_{d}$ (figure 7 ). The structure of the $\kappa_{e}\left(z_{*}\right)$ profile is particularly important as its curvature, i.e. $\partial^{2} \kappa_{e} / \partial z_{\star}^{2}$, determines if diapycnal mixing is 
able to 'sharpen' the local gradient. The sign of $\partial^{2} \kappa_{e} / \partial z_{*}^{2}$ could also provide a simple test for whether the mixing process is dominated by 'overturning' $\left(\partial^{2} \kappa_{e} / \partial z_{*}^{2}>0\right)$ or 'scouring' $\left(\partial^{2} \kappa_{e} / \partial z_{*}^{2}<0\right)$. Overturning-dominated mixing is reminiscent of the 'internal' mixing mechanism following the classification by Turner (1973). The turbulence which drives internal mixing occurs within the region where a large gradient of buoyancy is present. The 'external' mixing mechanism, however, is driven by turbulence external to the region with large gradient of buoyancy. It follows that the scouring processes examined here, which are critical in the maintenance of density interfaces, are 'external' in nature following Turner's terminology. When Richardson and Péclet numbers are both sufficiently large, we found the possibility of a density interface surviving due to the suppression of overturning shear instabilities by large Richardson number, and comparatively weak laminar diffusion at large Péclet number. Scouring by the external turbulence is key to the robustness of very stable 'sharp' interfaces. The framework employed in this analysis is effective for examining the spatial inhomogeneity of diapycnal mixing in the vertical direction and can be readily applied to investigate similar flows where layers and interfaces are the dominant features.

We have highlighted the relevance of molecular properties of the fluid (i.e. Prandtl number $P r$ ) in the 'right-flank' of Phillips' flux-gradient curve in determining the mixing properties of a sheared density interface (see e.g. figure 16), and this is critically because diapycnal transport does not vanish when the stratification is particularly strong and the molecular flux becomes important in such 'right-flank' situations. The kinetic energy available for mixing is supplied by vertical shear maintained by the walls in the layered stratified plane Couette (LSPC) flow configuration, and an important feature of this simple shear flow is the strong correlation between the gradient Richardson number and the buoyancy Reynolds number (as shown in figure 18(a)). When the gradient Richardson number is small, i.e. $R i_{g, *} \lesssim 0.25$, shear-induced overturns dominate in the $\mathrm{T}$ state of LSPC simulations, and the mixing efficiency is comparable to the data reported by Salehipour et al. (2016b) based on Kelvin-Helmholtz simulations (see figure 18(b)). The same observation applies when we compare the LSPC flow results to forced statistically stationary turbulence in the limit of large turbulent Froude number (weak stratification) $F r_{h, *} \gtrsim 1$, where the scaling $E \propto R i_{g, *} \propto F r_{h, *}^{-2}$ (see figure 19) seems to hold regardless of the forcing mechanism. However, turbulence cannot be sustained at large gradient Richardson numbers $\gtrsim 0.25$ in our LSPC flow configuration where the only forcing comes from vertical shear, and laminar diffusion immediately becomes relevant in determining the mixing properties for strongly stratified interfaces (see figure 16). This is in contrast to body-forced turbulence studies, e.g. Maffioli et al. (2016), where the flow stays energised under strong stratification by internal body forcing, and hence 'internal' mixing in the sense of Turner (1973). The mixing efficiency does not saturate to a constant, as is in standard turbulence parameterizations, e.g. Mellor \& Yamada (1982), in the limit of strong stratification, and molecular diffusivity does affect the mixing properties.

In this paper, we have investigated the self-sustaining mechanism of a sharp density interface when the Péclet number is sufficiently large, i.e. the external effects of the 'scouring' induced by the turbulence away from the interface and comparatively weak molecular diffusion across the core central region of the interface. It appears that a sharp density interface can be maintained by a subtle yet robust balance and interplay between molecular processes in the 'interface', where there is a strong density gradient suppressing vertical motions, and vigorous scouring turbulence in the much more weakly stratified 'layers' above and below the interface. This self-sustaining mechanism might explain how layers and interfaces may be robust structures in stably stratified geophysical flows, and this mechanism is intrinsically related to the mechanism proposed by Phillips (1972) 
regarding how these structures may form. On the other hand, we have only considered the 'robustness' of an existing density interface with a fixed initial thickness in this paper. Possible formation mechanisms of such layered structures from initially linearly stratified flows is the topic of a separate study (Taylor \& Zhou 2017).

The EPSRC Programme Grant EP/K034529/1 entitled 'Mathematical Underpinnings of Stratified Turbulence' is gratefully acknowledged for supporting the research presented here. We thank Professor S. G. Monismith for illuminating discussions and sharing postprocessed data from Shih et al. (2005) which are shown in figure 16(b). Dr A. Maffioli and $\mathrm{Mr} \mathrm{H}$. Salehipour are acknowledged for facilitating comparisons to their results on mixing efficiency presented in this paper. We thank three anonymous referees whose comments helped improve the manuscript significantly.

\section{REFERENCES}

Baines, P. G. \& Mitsudera, H. 1994 On the mechanism of shear flow instabilities. J. Fluid Mech. 276, 327-342.

Balmforth, N. J., Llewellyn-Smith, S. G. \& Young, W. R. 1998 Dynamics of interfaces and layers in a stratified turbulent fluid. J. Fluid Mech. 355, 329-358.

Bouffard, D. \& Boegman, L. 2013 A diapycnal diffusivity model for stratified environmental flows. Dyn. Atmos. Oceans 61-62, 14-34.

Brethouwer, G., Billant, P., Lindborg, E. \& Chomaz, J.-M. 2007 Scaling analysis and simulation of strongly stratified turbulent flows. J. Fluid Mech. 585, 343-368.

Carpenter, J. R., Balmforth, N. J. \& Lawrence, G. A. 2010 Identifying unstable modes in stratified shear layers. Phys. Fluids 22, 054104.

Carpenter, J. R., Tedford, E. W., Heifetz, E. \& Lawrence, G. A. 2011 Instability in stratified shear flow: Review of a physical interpretation based on interacting waves. Appl. Mech. Rev. 64, 060801.

Caulfield, C.-C. P. 1994 Multiple instability of layered stratified shear flow. J. Fluid Mech. 258, 255-285.

Caulfield, C. P. \& Peltier, W. R. 2000 The anatomy of the mixing transition in homogeneous and stratified free shear layers. J. Fluid Mech. 413, 1-47.

Crapper, P. F. \& Linden, P. F. 1974 The structure of turbulent density interfaces. J. Fluid Mech. 65, 45-63.

Deusebio, E., Caulfield, C. P. \& Taylor, J. R. 2015 The intermittency boundary in stratified plane Couette flow. J. Fluid Mech. 781, 298-329.

Eaves, T.S. \& CAulfield, C. P. 2017 Multiple instability of layered stratified plane Couette flow. J. Fluid Mech. 813, 250-278.

Eliassen, A., Hailand, E. \& RiIs, E. 1953 Two-dimensional perturbation of a flow with constant shear of a stratified fluid. Inst. Weather Climate Res., Norwegian Acad. Sci. Lett. Publ. no. 1.

Fernando, H. J. S. 1991 Turbulent mixing in stratified fluids. Annu. Rev. Fluid Mech. 23, 455-493.

Gregg, M.C. 1980 Microstructure patches in the thermocline. J. Phys. Oceanogr. 10, 915-943.

Linden, P. F. 1979 Mixing in stratified fluids. Geophys. Astro. Fluid Dyn. 13, 3-23.

Maffioli, A., Brethoumer, G. \& Lindborg, E. 2016 Mixing efficiency in stratified turbulence. J. Fluid Mech. 794, R3.

Marshall, J., Shuckburgh, E., Jones, H. \& Hill, C. 2006 Estimates and implications of surface eddy diffusivity in the southern ocean derived from tracer transport. J. Phys. Oceanogr. 36, 1806-1821.

Mashayek, A., Caulfield, C. P. \& Peltier, W. R. 2013 Time-dependent, non-monotonic mixing in stratified turbulent shear flows: implications for oceanographic estimates of buoyancy flux. J. Fluid Mech. 736, 570-593.

Mellor, G. L. \& YAMAdA, T. 1982 Development of a turbulence closure model for geophysical fluid problems. Rev. Geophys. 20, 851-875. 
NAKAmura, N. 1996 Two-dimensional mixing, edge formation, and permeability diagnosed in an area coordinate. J. Atoms. Sci. 53, 1524-1537.

Oglethorpe, R. L. F., Caulfield, C. P. \& Woods, A. W. 2013 Spontaneous layering in stratified turbulent Taylor-Couette flow. J. Fluid Mech. 721, R3.

Peltier, W. R. \& Caulfield, C. P. 2003 Mixing efficiency in stratified shear flows. Annu. Rev. Fluid Mech. 35, 135-167.

Phillips, O. M. 1972 Turbulence in a strongly stratified fluid - is it unstable? Deep-Sea Res. 19, $79-81$.

Pope, S. B. 2000 Turbulent Flows. Cambridge University Press.

Posmentier, E. S. 1977 The generation of salinity fine structure by vertical diffusion. J. Phys. Oceanogr. 7, 298-300.

Ruddick, B.R., McDougall, T.J. \& Turner, J.S. 1989 The formation of layers in a uniformly stirred density gradient. Deep-Sea Res. 36, 597-609.

Salehipour, H., Caulfield, C. P. \& Peltier, W.R. 2016a Turbulent mixing due to the Holmboe wave instability at high Reynolds number. J. Fluid Mech. 803, 591-621.

Salehipour, H., Peltier, W.R., Whalen, C.B. \& Mackinnon, J.A. $2016 b$ A new characterization of the turbulent diapycnal diffusivities of mass and momentum in the ocean. Geophys. Res. Lett. 43, 3370-3379.

Salehipour, H. \& Peltier, W. R. 2015 Diapycnal diffusivity, turbulent Prandtl number and mixing efficiency in Boussinesq stratified turbulence. J. Fluid Mech. 775, 464-500.

Shin, L. H., Koseff, J. R., Ivey, G. N. \& Ferziger, J. H. 2005 Parameterization of turbulent fluxes and scales using homogeneous sheared stably stratified turbulence simulations. $J$. Fluid Mech. 525, 193-214.

Shuckburgh, E. \& Haynes, P. 2003 Diagnosing transport and mixing using a tracer-based coordinate system. Phys. Fluids 15, 3342-3357.

Smyth, W.D., Klatassen, G.P. \& Peltier, W.R. 1988 Finite amplitude Holmboe waves. Geophys. Astro. Fluid Dyn. 43, 181-222.

Smyth, W.D., Moum, J.N. \& NAsh, J.D. 2011 Narrowband oscillations in the upper equatorial ocean. Part II: Properties of shear instabilities. J. Phys. Oceanogr. 41, 412-428.

Smyth, W. D., Moum, J. \& Caldwell, D. 2001 The efficiency of mixing in turbulent patches: inferences from direct simulations and microstructure observations. J. Phys. Oceanogr. 31, 1969-1992.

Strang, E. J. \& Fernando, H. J. S. 2001 Entrainment and mixing in stratified shear flows. J. Fluid Mech. 428, 349-386.

TAYlor, J. R. 2008 Numerical Simulations of the Stratified Oceanic Bottom Boundary Layer. $\mathrm{PhD}$ thesis, University of California, San Diego.

TAYloR, J. R. \& Zhou, Q. 2017 A multi-parameter criterion for layer formation in a stratified shear flow using buoyancy coordinates. Submitted to J. Fluid Mech.

Thorpe, S. A. 2005 The Turbulent Ocean. Cambridge University Press.

Thorpe, S. A. 2016 Layers and internal waves in uniformly stratified fluids stirred by vertical grids. J. Fluid Mech. 793, 380-413.

Tseng, Y. \& Ferziger, J. H. 2001 Mixing and available potential energy in stratified flows. Phys. Fluids 13, 1281-1293.

Turner, J. S. 1973 Buoyancy Effects in Fluids. Cambridge University Press.

Venaille, A., Gostiaux, L. \& Sommeria, J. 2017 A statistical mechanics approach of mixing in stratified fluids. J. Fluid Mech. 810, 554-583.

Venayagamoorthy, S. K. \& Koseff, J. R. 2016 On the flux Richardson number in stably stratified turbulence. J. Fluid Mech. 798, R1.

Winters, K. B. \& D'Asaro, E. A. 1996 Diascalar flux and the rate of fluid mixing. J. Fluid Mech. 317, 179-193.

Winters, K. B., Lombard, P. N., Riley, J. J. \& D'Asaro, E. A. 1995 Available potential energy and mixing in density-stratified fluids. J. Fluid Mech. 289, 115-128.

Woods, A. W., Caulfield, C. P., Landel, J. R. \& Kuesters, A. 2010 Non-invasive turbulent mixing across a density interface in a turbulent Taylor-Couette flow. J. Fluid Mech. 663, 347-357.

Zhou, Q., Taylor, J. R. \& Caulfield, C. P. 2017 Self-similar mixing in stratified plane couette flow for varying Prandtl number. Accepted for publication in J. Fluid Mech. 